\title{
Status Report on the Modeling of TRISO Energy Deposition, Time-Dependent Temperature Field and Doppler Feedback
}

Javier Ortensi

Abderrafi M. Ougouag

July 2009
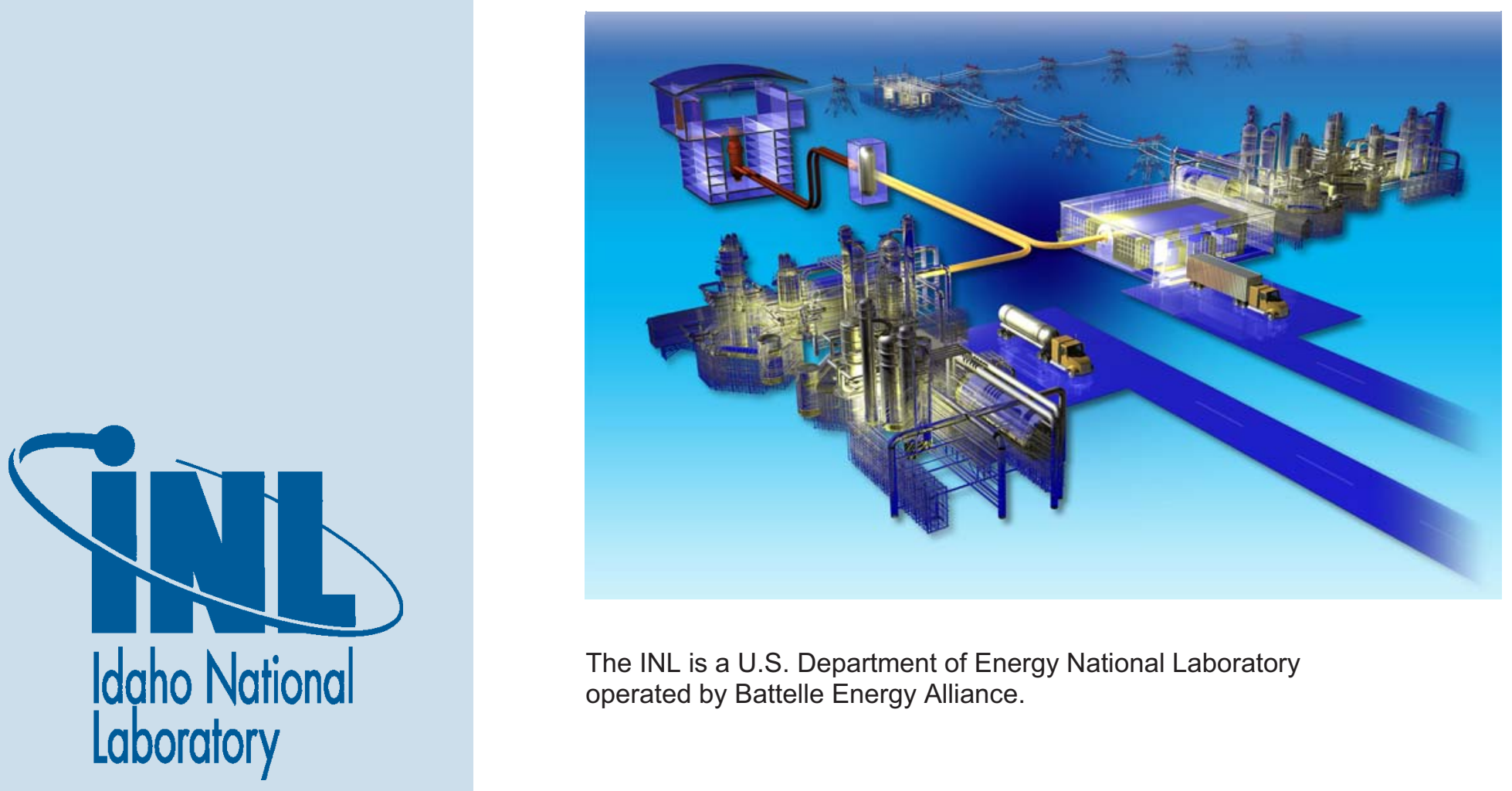

The INL is a U.S. Department of Energy National Laboratory operated by Battelle Energy Alliance. 


\section{DISCLAIMER}

This information was prepared as an account of work sponsored by an agency of the U.S. Government. Neither the U.S. Government nor any agency thereof, nor any of their employees, makes any warranty, expressed or implied, or assumes any legal liability or responsibility for the accuracy, completeness, or usefulness, of any information, apparatus, product, or process disclosed, or represents that its use would not infringe privately owned rights. References herein to any specific commercial product, process, or service by trade name, trade mark, manufacturer, or otherwise, does not necessarily constitute or imply its endorsement, recommendation, or favoring by the U.S. Government or any agency thereof. The views and opinions of authors expressed herein do not necessarily state or reflect those of the U.S. Government or any agency thereof. 


\title{
Status Report on the Modeling of TRISO Energy Deposition, Time-Dependent Temperature Field and Doppler Feedback
}

\author{
Javier Ortensi \\ Abderrafi M. Ougouag
}

July 2009

\begin{abstract}
Idaho National Laboratory
Next Generation Nuclear Plant Project

Idaho Falls, Idaho 83415
\end{abstract}

Prepared for the

U.S. Department of Energy

Office of Nuclear Energy

Under DOE Idaho Operations Office

Contract DE-AC07-05ID14517 

Next Generation Nuclear Plant Project

\section{Status Report on the Modeling of TRISO Energy Deposition, Time-Dependent Temperature Field and Doppler Feedback}

INL/EXT-09-16410

July 2009

Approved by:

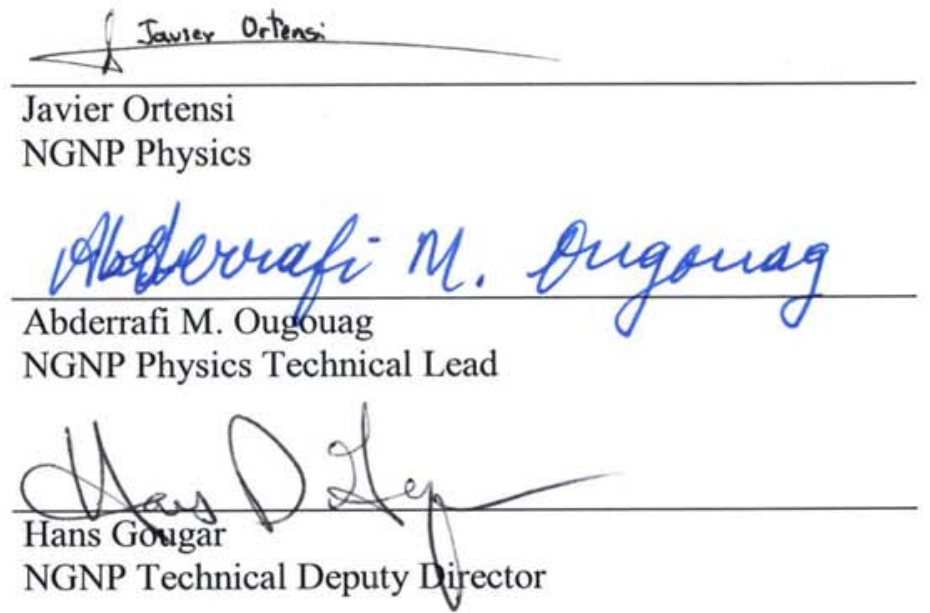

\section{$7 / 23 / 2009$} Date
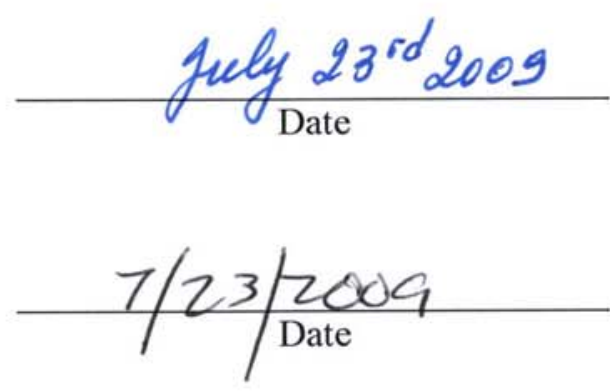



\begin{abstract}
The Doppler feedback mechanism is a major contributor to the passive safety of gas-cooled, graphite-moderated high-temperature reactors that use fuel-based Tristructural-Isotropic (TRISO) coated particles. It follows that the correct prediction of the magnitude and time dependence of this feedback effect is essential to the conduct of safety analyses for these reactors. We present a fuel conduction model for obtaining better estimates of the temperature feedback during moderate and fast transients. The fuel model has been incorporated in the CYNOD-THERMIX-KONVEK suite of coupled codes as a single TRISO particle within each calculation cell. The heat generation rate is scaled down from the neutronic solution and a Dirichlet boundary condition is imposed as the bulk graphite temperature from the thermal-hydraulic solution. This simplified approach yields similar results to those obtained with more complex methods, requiring multi-TRISO calculations within one control volume, but with much less computational effort. An analysis of the hypothetical total control ejection event in the pebble bed modular reactor (PBMR)-400 design that clearly depicts improvement in fuel temperature predictions is provided.
\end{abstract}




\section{ACKNOWLEDGEMENTS}

Work supported by the U.S. Department of Energy, Assistant Secretary for the office of Nuclear Energy, under DOE Idaho Operations Office Contract DEAC07-05ID14517. 


\section{CONTENTS}

ABSTRACT vi

ACKNOWLEDGEMENTS viii

ACRONYMS xii

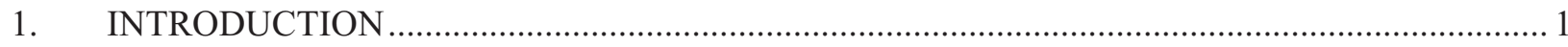

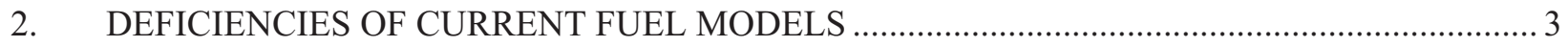

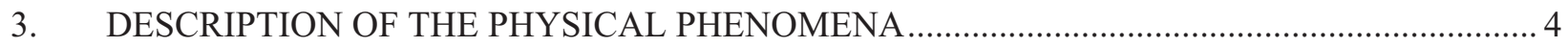

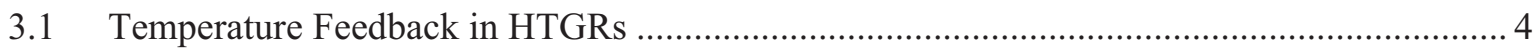

Effects from Thermo-Physical Properties ......................................................................... 4

4. DESCRIPTION OF THE MATHEMATICAL MODELS AND NUMERICAL

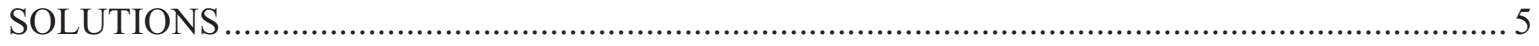

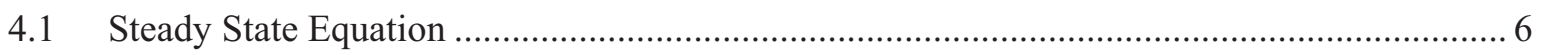

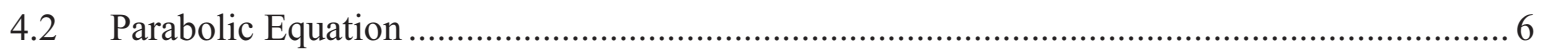

5. VERIFICATION OF THE NUMERICAL MODEL …........................................................... 7

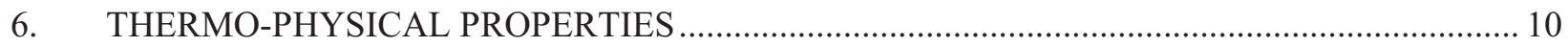

7. INTEGRATION INTO THE COUPLED NEUTRONIC-TH SYSTEM ....................................... 11

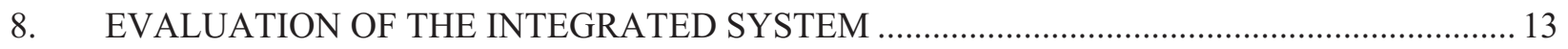

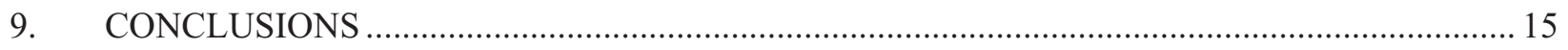

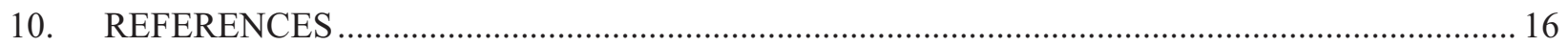

Appendix A-Analytic Solution to the 1-D Spherical Steady State Heat Conduction Equation ............... 17

Appendix B-Numerical Solution to the 1-D Spherical Parabolic Heat Conduction Equation ................. 21

Appendix C-Analytic Solution to the 1-D Spherical Parabolic Heat Conduction Equation ....................27

\section{FIGURES}

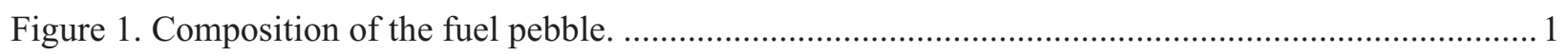

Figure 2. Homogeneous and explicit models for a HTGR fuel pebble.................................................... 3

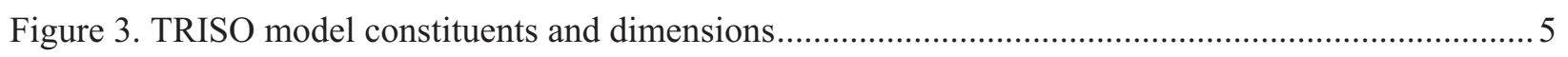

Figure 4. Benchmark with constant thermo-physical properties and constant heat generation. .................. 7

Figure 5. Benchmark with constant thermo-physical properties linear heat generation............................. 8

Figure 6. Benchmark with constant thermo-physical properties exponential heat generation. ................... 8 
Figure 7. Benchmark with variable thermo-physical properties - Temperatures. .

Figure 8 . Benchmark with variable thermo-physical properties - Percent difference in

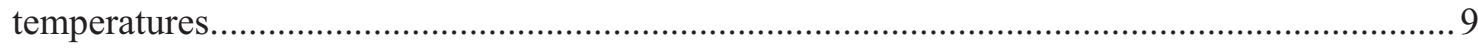

Figure 9. Thermal conductivities for the TRISO constituent layers. ................................................... 10

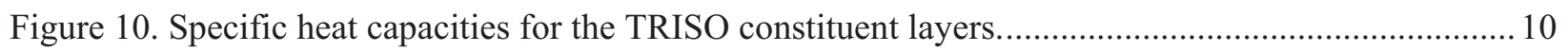

Figure 11. Coupling between the neutronic code CYNOD and thermal-hydraulics THERMIX-

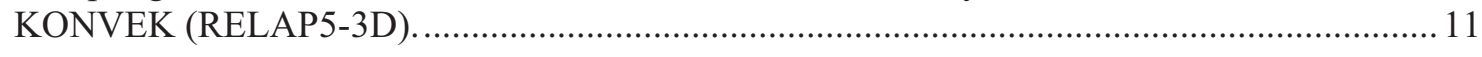

Figure 12. Relative reactor total power during the TCRE transient..................................................... 13

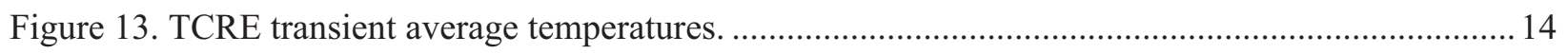




\section{ACRONYMS}

CEA Commissariat à l'Énergie Atomique

DBA Design Basis Accident

DOE Department of Energy

HFP Hot Full Power

HTGR High Temperature Gas-Cooled Reactor

HTR High Temperature Reactor

HZP Hot Zero Power

INL Idaho National Laboratory

KAIST Korea Advanced Institute of Science and Technology

NGNP Next-Generation Nuclear Plant

PBMR Pebble Bed Modular Reactor

PBR Pebble Bed Reactor

PLOFC Pressurized Loss of Forced Cooling

QSHe Quasi-Static Heterogeneous

QSHo Quasi-Static Homogeneous

SAR Safety Analysis Report

TCRE Total Control Rod Ejection

TDHe Time Dependent Heterogeneous

TRISO Tristructural-Isotropic 


\section{Status Report on the Modeling of TRISO Energy Deposition, Time-Dependent Temperature Field and Doppler Feedback}

\section{INTRODUCTION}

Doppler, moderator, and reflector feedback are three essential temperature feedback mechanisms in gas-cooled, graphite-moderated high temperature reactors (HTRs). These feedback effects provide the necessary passive safety for successful operation of these reactors. During moderately fast and fast reactor transients, the Doppler feedback mechanism is the dominating process that ensures this passive safety. Because the extent of the power escalation during these transients is determined by the fuel temperature, it is crucial to obtain accurate UO2 temperatures. Furthermore, the extent of the power escalation has an effect on the total energy deposition.

Modern designs of HTR cores are fueled with Tristructural-Isotropic (TRISO) coated particles. These particles are comprised of various layers acting in concert to provide a containment structure that prevents radioactive product release. For a pebble bed reactor (PBR), the TRISO particles are imbedded in a graphite matrix to form a pebble, as shown in Figure 1(OECD/NEA/NSC PBMR400 Benchmark 2007). The same particles are also used in the prismatic design by forming fuel compacts in a cylindrical graphite matrix. The TRISO particles include a fuel kernel, porous carbon layer, inner pyrolitic carbon (IPyC), SiC, and outer pyrolitic carbon (OPyC). The main functions of the various layers are heat generation in the kernel, fission product retention in the porous layer, structural integrity in both pyrolitic carbons, and fission product barrier in the $\mathrm{SiC}$. The heat transfer characteristics of these components determine the ultimate temperature of the $\mathrm{UO}_{2}$ fuel kernel. The various materials that constitute TRISO particles have different thermo-physical and thermo-mechanical properties with varied dynamic behavior. Therefore, any improvement to the heat transfer model results in a more accurate calculation of the fuel temperature and the reactor power.

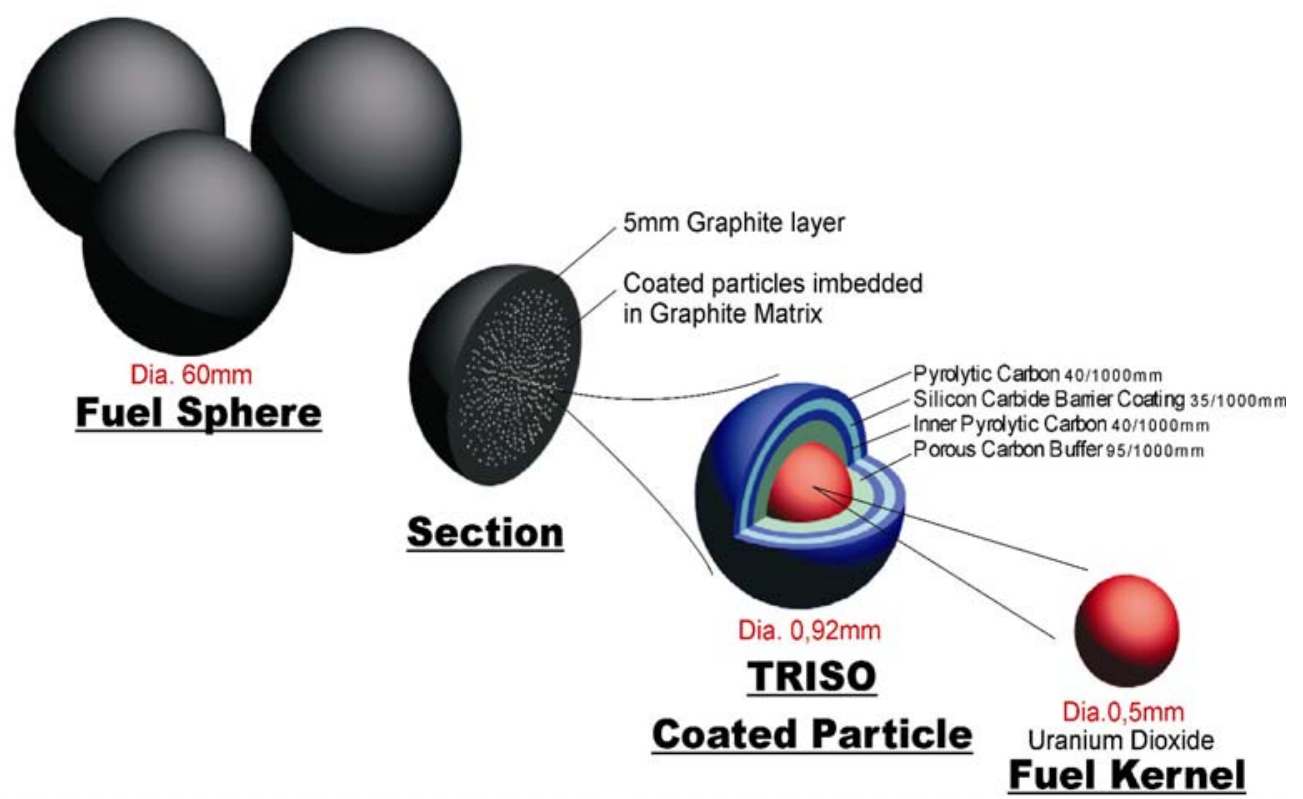

Figure 1. Composition of the fuel pebble. 
Most high temperature gas-cooled reactor (HTGR) codes, like THERMIX (Teuchert 1994), only include a homogeneous model, which approximates the temperature of the fuel region. These models produce reasonable results for steady-state and very slow transient conditions. For moderate and fast transients, they inadequately capture prompt effects. An alternative fuel model used in TINTE extrapolates the maximum fuel temperature from the solution to a homogenous model (Scherer 1989). The only HTR transient code before 2008 that appears to contain a fully explicit TRISO model is ZKIND (Rademer 2004). Unfortunately, no details on the development of ZKIND are available.

During the development of this work several articles have been published that specifically deal with explicit TRISO models. Noteworthy are the two-temperature models developed at KAIST (Cho 2009), the multi-scale methods from CEA (Blanc 2008) and AMEC (Stainsby 2008), and finally the modifications to TINTE by PBMR Ltd. (Ubbink 2008). Of these works, only TINTE has been successfully used in full reactor transient analysis (Strydom 2008). 


\section{DEFICIENCIES OF CURRENT FUEL MODELS}

The current Idaho National Laboratory (INL) coupled neutronics-thermal-hydraulics code CYNODTHERMIX-KONVEK (Hiruta 2008) includes a shell model that approximates the temperature of the fuel region. Figure 2a includes an illustration for pebble bed fuel. Consequently, the fuel region, which contains a mixture of fuel and graphite matrix, is treated as a homogeneous domain with constant volumetric heat generation. This type of model overlooks the fact that the majority of the fission energy is deposited in the fuel kernel and that there can be a strong resistance to heat flow within the TRISO constituents. In addition, by not modeling the particles explicitly, it is assumed that the various materials in the fuel exhibit similar thermal inertia and play a small role in the dynamic behavior of the bulk macroscopic material. These models produce reasonable results for steady-state and very slow transient conditions where these assumptions are valid. For moderate and fast transients, these homogenous models underestimate the temperature in the fuel kernel and, consequently, significantly overestimate the neutron flux and power level.

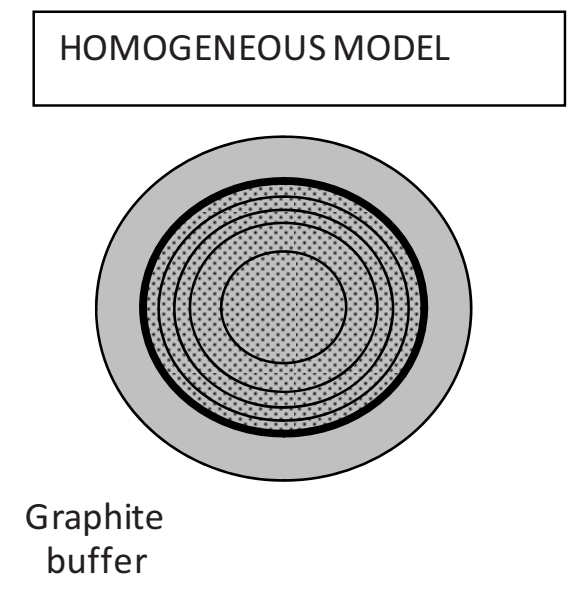

Fuel region

(2a)

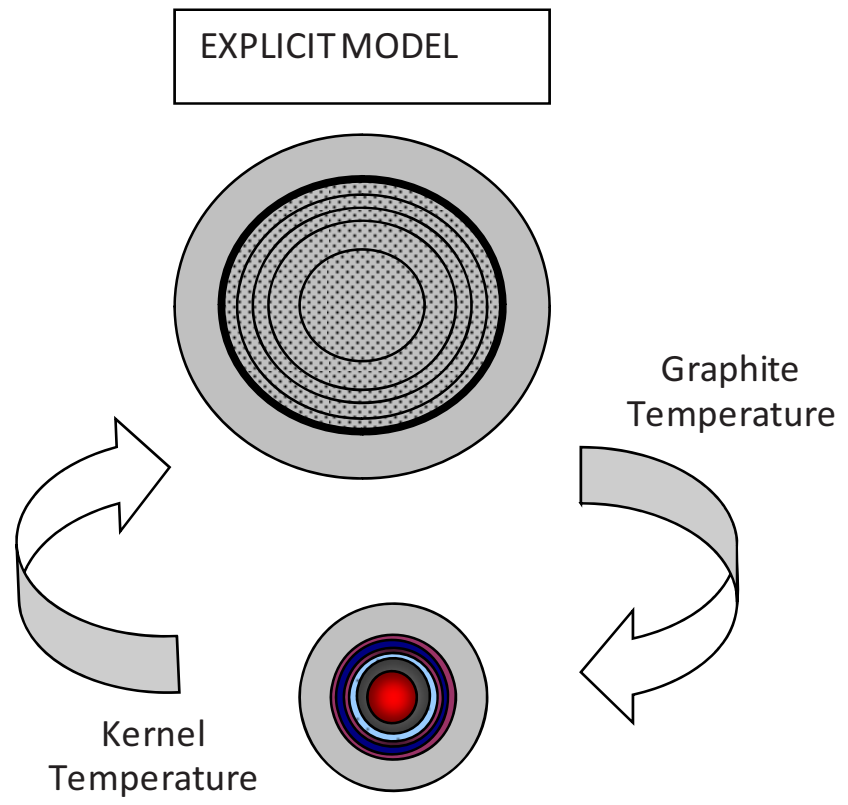

(2b)

Figure 2. Homogeneous and explicit models for a HTGR fuel pebble.

An explicit model, similar to the one depicted in Figure 2b, is necessary in order to accurately predict the fuel temperature in TRISO-fueled reactors. As depicted in the illustration, the explicit model uses the results from the homogeneous thermal-hydraulics calculation in order to determine the micro-scale heat transfer in the TRISO particle. 


\section{DESCRIPTION OF THE PHYSICAL PHENOMENA}

Nuclear fuel at steady state is in quasi-thermal equilibrium, where the reactor is kept in a quasicritical state by feedback mechanisms. Large departures from this quasi-equilibrium state are encountered when the neutron distribution changes significantly due to some material or geometrical modification of the system, which can arise from temperature changes or other means.

The effect that temperature changes have on the dynamic response of a nuclear reactor is commonly known as temperature feedback. A number of important physical phenomena determine the magnitude of temperature feedback. In HTRs, these phenomena are captured by essentially three feedback mechanisms: Doppler, moderator, and reflector.

\subsection{Temperature Feedback in HTGRs}

The Doppler temperature feedback is a stabilizing, almost instantaneous effect in fuel. This temperature feedback is the consequence of Doppler broadening, a neutronic phenomenon driven by changes in the relative velocities of the U-238 atoms and the neutrons and has a direct effect on the neutron population through resonance absorption. These atom velocities are represented by the macroscopic material temperatures. Therefore, changes to the fuel temperature affect the magnitude of Doppler feedback, which, in turn, modifies the neutron population and, thus, the power generation.

The moderator temperature feedback effect is due to the spectral shifting induced by scattering in graphite and by a reduction in absorption of thermal neutrons in graphite at higher temperatures. It is normally a stabilizing effect, but can, in the presence of some low-lying resonances, be de-stabilizing in nature. It is a delayed effect, when compared to the Doppler temperature feedback.

The reflector temperature feedback effect is a de-stabilizing mechanism in graphite moderated reactors. It is a largely delayed effect since the heat-up of the reflector region is a slow process during transients. The effect emerges from the reduced absorption of thermal neutrons in graphite at higher temperatures, which allows more thermal neutrons to enter the reactor core.

\subsection{Effects from Thermo-Physical Properties}

The various materials that constitute TRISO particles and the rest of core and reflector materials have different thermo-physical and thermo-mechanical properties with varied dynamic behavior. From a heat transfer perspective, the layered composition of the TRISO generates a natural resistance to heat flow. This effect was previously identified (Merrill 1963), but the explicit modeling of the fuel particles was never pursued, as far as the authors know, due to the lack of data for the heat transfer model.

The Doppler feedback is pronounced in HTRs mainly due to the relatively low heat capacity of $\mathrm{UO}_{2}$ when compared to the surrounding constituents of TRISO fuel. In addition, the majority of the energy deposition occurs in the fuel kernel as well. This allows the kernel temperature to increase significantly faster that its surroundings, a fact that is overlooked in homogenous models.

The delayed nature of both the moderator and reflector feedbacks are mainly due to the large volumetric heat capacity of graphite, which effectively delays the temperature rise in the core graphite and surrounding reflector. Since the nuclear phenomena of absorption and scattering of neutrons is highly sensitive to material temperatures, this delay is equally experienced in the changes to the neutron population. 


\section{DESCRIPTION OF THE MATHEMATICAL MODELS AND NUMERICAL SOLUTIONS}

The approach developed in this work incorporates an explicit TRISO heat conduction model within the framework of the solution to the homogeneous fuel model attained via a thermal-hydraulic code. Figure 3 shows the materials and dimensions used in modeling the TRISO fuel particle.

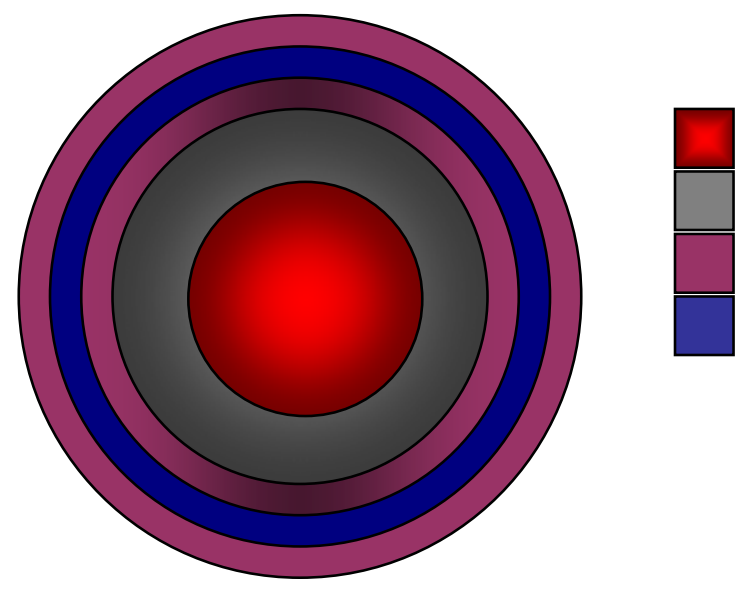

\section{Material / layer thickness}

$\mathrm{UO}_{2} /(250 \mu \mathrm{m})$

Porous Carbon / (95 $\mu \mathrm{m})$

Pyrolitic Carbon / $(40 \mu \mathrm{m})$

$\mathrm{SiC} /(35 \mu \mathrm{m})$

Figure 3. TRISO model constituents and dimensions

A one-dimensional (1-D) geometrical model is used in this work. This assumes angular symmetry, i.e. heat conduction out of the TRISO particle only varies in the radial direction. The one dimensional PDE that governs heat conduction is:

$$
\nabla \cdot(k(r, t) \nabla T(r, t))+q^{\prime \prime}(r, t)=\frac{\partial \rho(r, t) C_{p}(r, t) T(r, t)}{\partial t} 0 \leq r \leq R \text { for } \mathrm{t} \geq 0
$$

where $\mathrm{k}, \rho$, and $\mathrm{C}_{\mathrm{p}}$ are the thermal conductivity, density, and specific heat capacity, respectively. In order to simplify the problem, it is assumed that the thermo-physical properties do not vary significantly in between time steps, thus yielding the following:

$$
\nabla \cdot(k(r) \nabla T(r, t))+q^{\prime \prime}(r, t)=\rho(r) C_{p}(r) \frac{\partial T(r, t)}{\partial t} \quad 0 \leq r \leq R \text { for } \mathrm{t} \geq 0
$$

This implies an error in the energy conservation that is very small in magnitude when compared to the changes in temperature. This small error can be further reduced by performing nonlinear iterations to update the thermo-physical properties. The solution to this second order PDE requires two boundary conditions and one initial condition. The boundary conditions imposed are a Neumann ( $2^{\text {nd }}$ kind) condition at the center and Dirichlet $\left(1^{\text {st }}\right.$ kind $)$ at the outer surface:

$\left.\frac{\partial T(r, t)}{\partial r}\right|_{r=0}=\left.0 T(r, t)\right|_{\partial D}=T_{g}(t)$ for $\mathrm{t} \geq 0$

The initial condition will be the steady-state temperature distribution at the initial time:

$$
T(r, 0)=T_{s s}(r)
$$




\subsection{Steady State Equation}

Equation 2 can be further simplified by eliminating the time dependence and assuming a homogeneous thermal conductivity yielding the 1-D spherical steady-state heat conduction equation

$\frac{d^{2} T}{d r^{2}}+\frac{2}{r} \frac{d T}{d r}+\frac{q^{\prime \prime}}{k}=0 \quad 0 \leq r \leq R$

The derivation of the solution to this steady state problem can be found in Appendix A. The temperature field for heated region is given by

$$
T_{\text {heated }}(r)=\frac{q_{i}^{\prime \prime \prime}}{6 k_{i}}\left(r_{i+1}^{2}-r^{2}\right)+T_{i+1}
$$

The temperature field in non-heated regions is obtained by imposing to Equation A-1 a nonzero heat flux at the lower boundary, $-\left.k_{1} \frac{d T}{d r}\right|_{r=r_{i}}=\frac{r_{1} q_{1}^{\prime \prime}}{3}$, yielding

$$
T_{\text {unheated }}(r)=\frac{r_{f s}^{3} q^{\prime \prime}}{3 k_{i}}\left(\frac{1}{r}-\frac{1}{r_{i-1}}\right)+T_{i-1}
$$

\subsection{Parabolic Equation}

Equation 2 can be rewritten in the heat flux formulation via Fourier's law:

$$
-\nabla \cdot \vec{q}(r, t)+q " \prime(r, t)=\rho(r) C_{p}(r) \frac{\partial T(r, t)}{\partial t} \quad 0 \leq r \leq R \text { for } \mathrm{t} \geq 0
$$

Appendix B contains the derivation for the numerical solution to this equation. Employing a theta time differencing scheme, using Fourier's law as the closure model, and forcing the continuity of the heat flux at the interfaces yields a three-point formulation with the average cell temperatures.

$$
\begin{aligned}
& \theta\left(a_{i+1} \widetilde{T}_{i+1}^{n+1}+a_{i} \widetilde{T}_{i}^{n+1}+a_{i-1} \widetilde{T}_{i-1}^{n+1}\right)=\left((1-\theta) q_{i}^{\prime, n}+\theta q_{i}^{\prime,{ }^{n+1}}\right) V_{i} \\
& +(1-\theta)\left(b_{i+1} \widetilde{T}_{i+1}^{n}+b \widetilde{T}_{i}^{n}+b_{i-1} \widetilde{T}_{i-1}^{n}\right)
\end{aligned}
$$

Since the final matrix is positive, definite, tri-diagonal, and symmetric, the direct inversion of the two-banded system of equations yields the final solution. 


\section{VERIFICATION OF THE NUMERICAL MODEL}

The numerical solution to the time-dependent equation developed in Section 4.2 was implemented into a FORTRAN 90 computer code and is referred to in this work as the Time Dependent Heterogeneous TDHe model. The results from this program are benchmarked against the analytic solution to the 1-D spherical time-dependent conduction equation developed in Appendix C. The model used in the test is a sphere of UO2 with $0.042-\mathrm{cm}$ radius initially at $917 \mathrm{~K}$. In addition, the code is also compared to the finite difference code HEATING (Childs 2007). Similar spatial and time discretizations are used to generate consistent comparisons of the computer implementations.

Figure 4 shows the percent difference in the temperature calculations for a homogeneous sphere with constant thermo-physical properties and a constant heat generation rate. The small differences, in the order of $0.006 \%$, seen in HEATING 7.3 appear to arise from the solution methodology. The TDHe results are in excellent agreement with the analytic solution on HEATING.

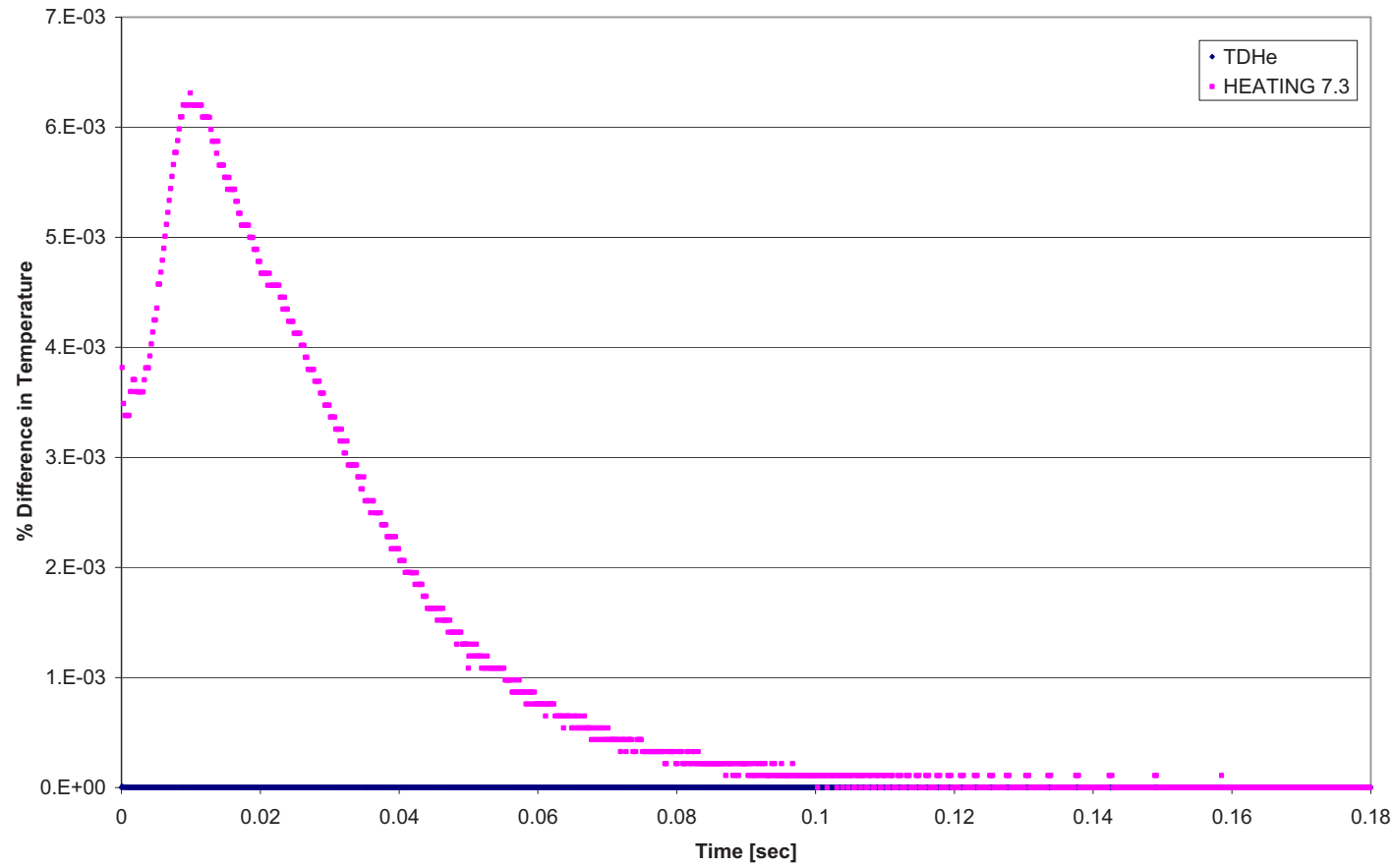

Figure 4. Benchmark with constant thermo-physical properties and constant heat generation.

The next test is used to study the solutions with a linear heat generation rate. Figure 5 shows that the TDHe results are also in good agreement with the rest of the solutions. The HEATING results reach a calculation precision limit of $1 \mathrm{E}-3 \%$ for this case with linear heat generation rate. 


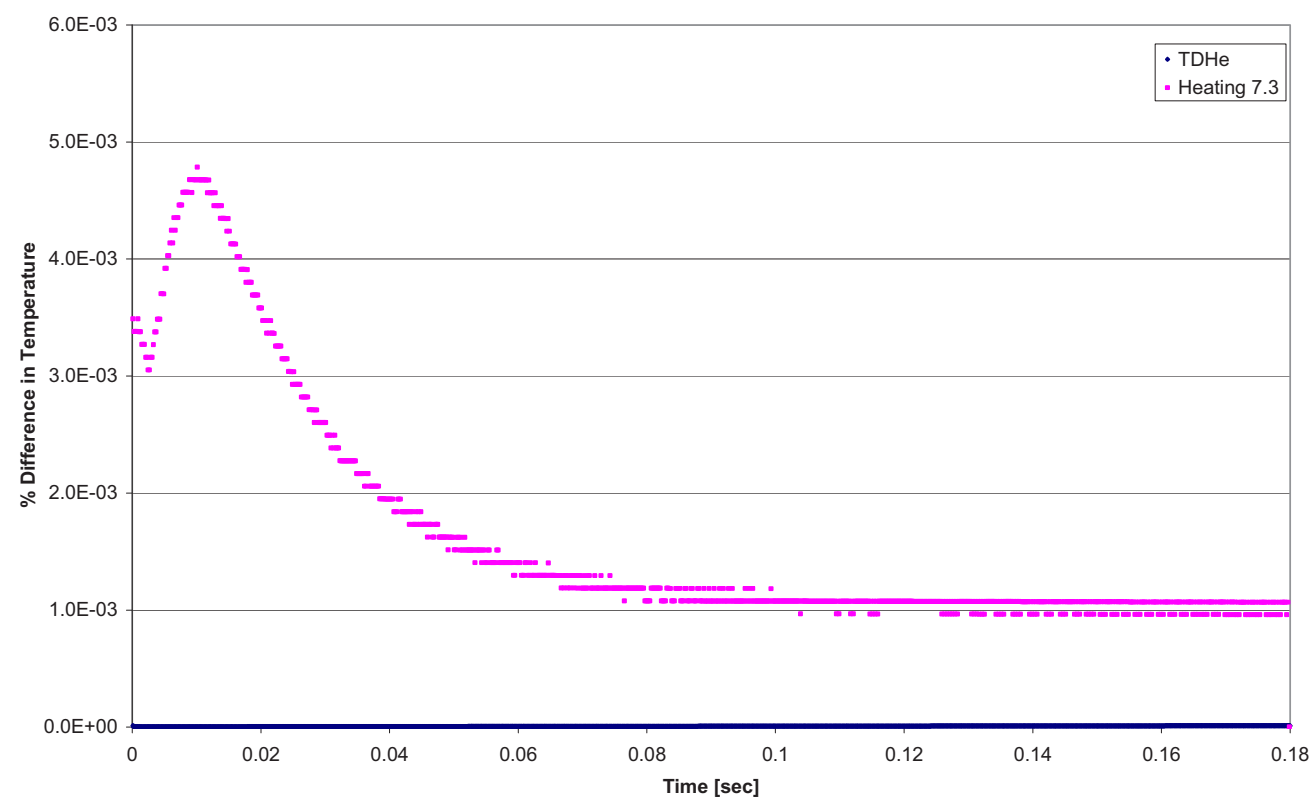

Figure 5. Benchmark with constant thermo-physical properties linear heat generation.

Figure 6 shows the percent difference in the temperature calculations for a homogeneous sphere with constant thermo-physical properties and an exponential heat generation rate. Again, very good agreement is obtained between the TDHe and the analytic solution and the same pattern is observed for the percent errors from HEATING.

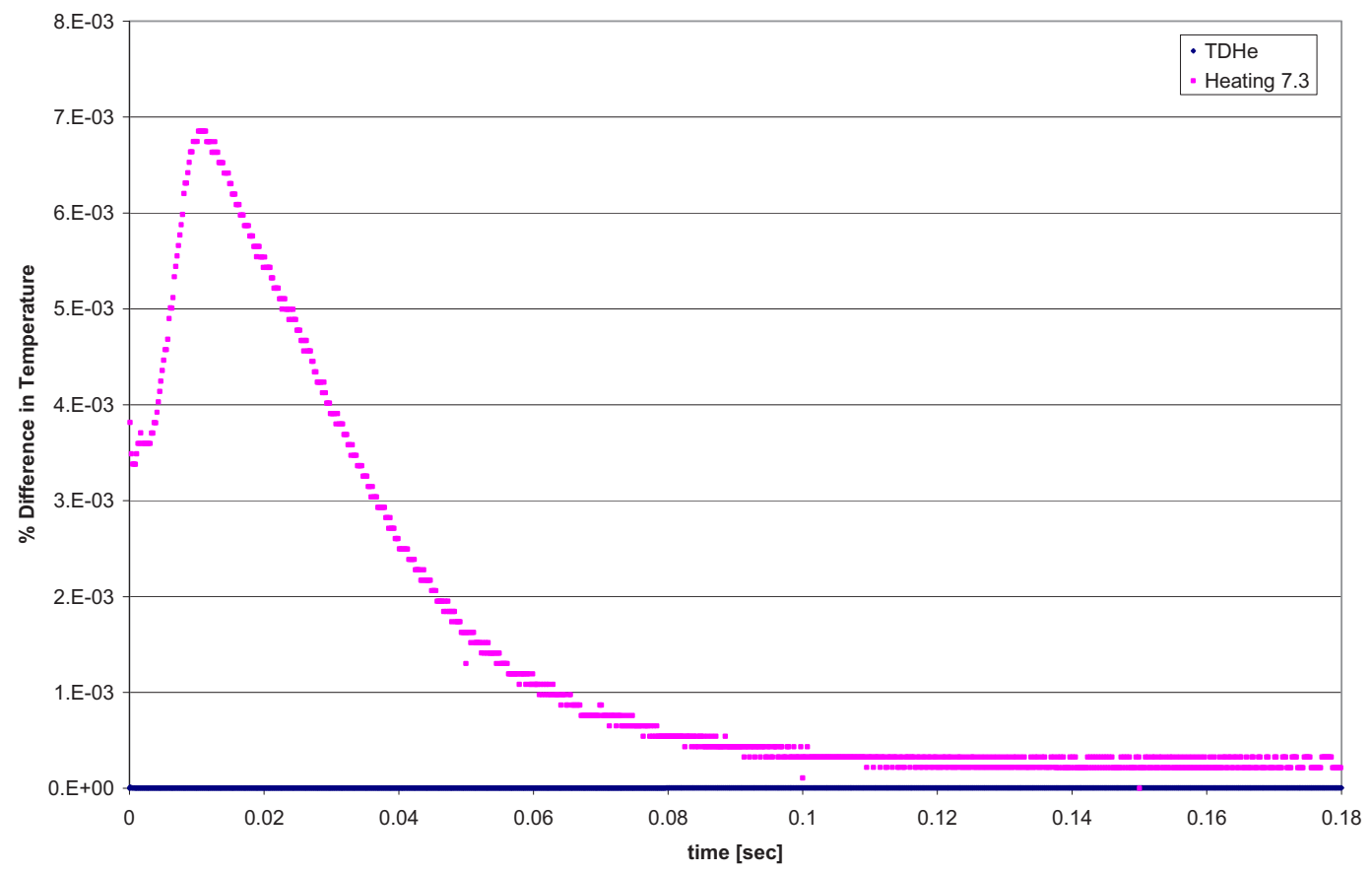

Figure 6. Benchmark with constant thermo-physical properties exponential heat generation. 
A code-to-code comparison is used to benchmark the nonlinear solution, since no analytic solution is available for the nonlinear case. Figures 7 and 8 present the comparison to HEATING for a model with variable properties in all of the layers and for an assumed power excursion. The code comparisons agree with maximum differences of $0.06 \%$.

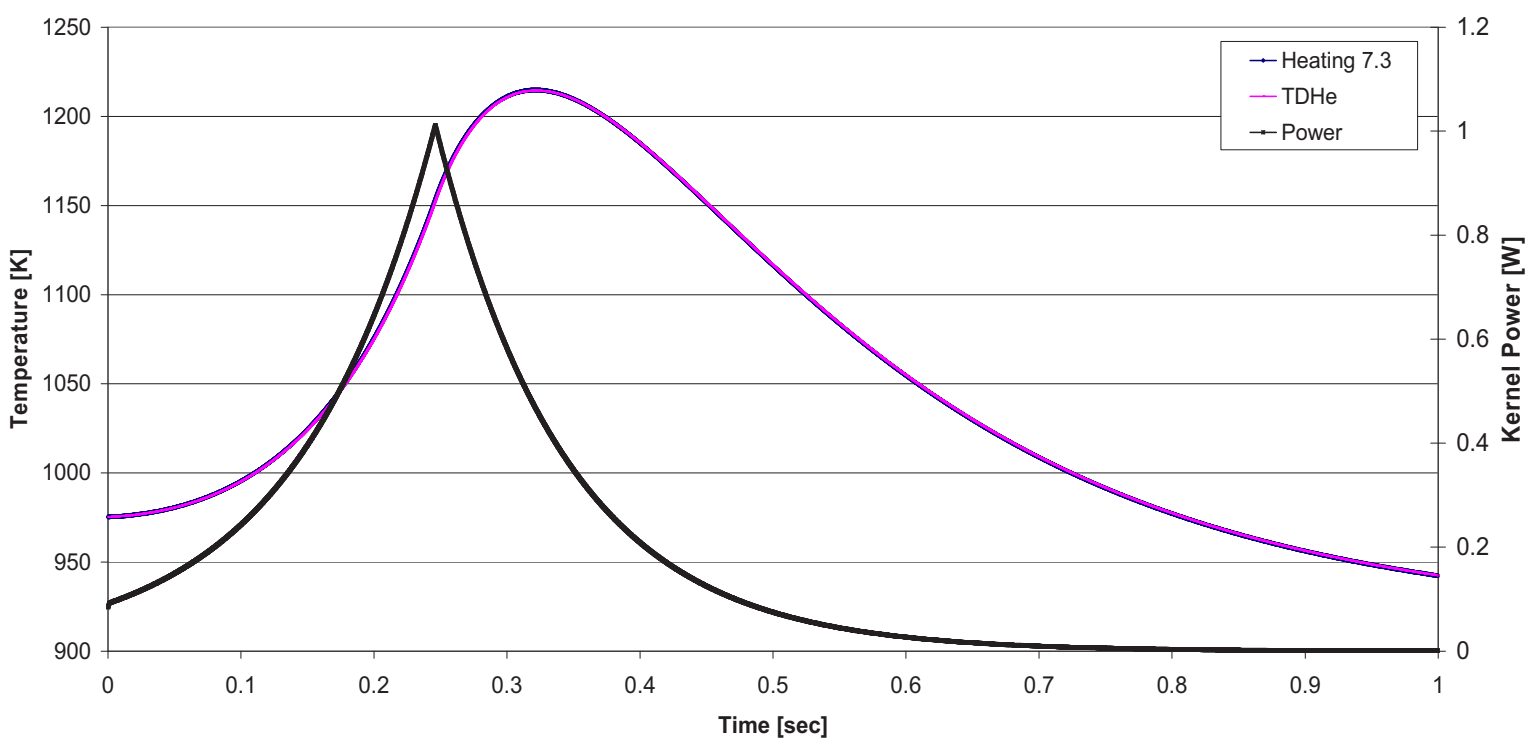

Figure 7. Benchmark with variable thermo-physical properties - Temperatures.

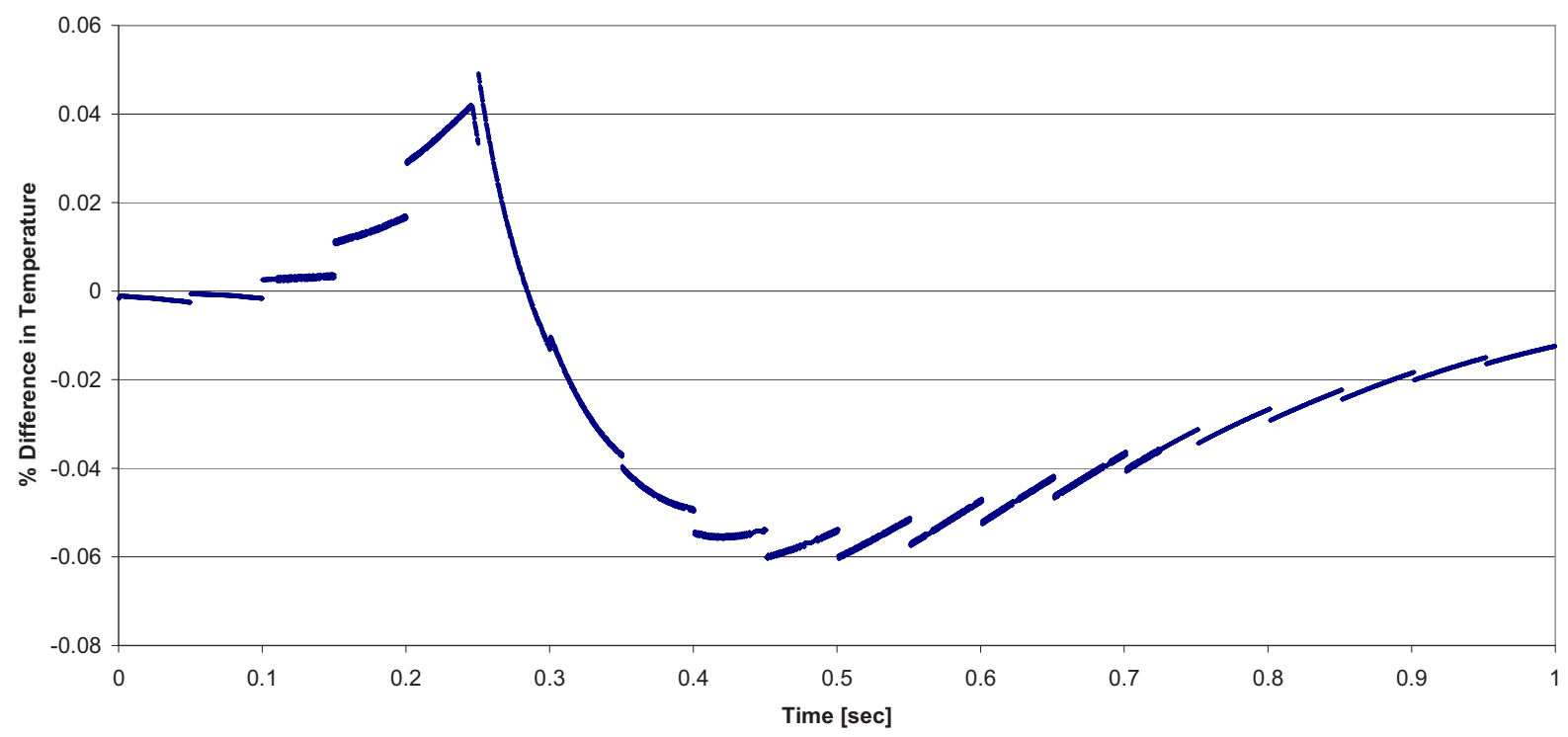

Figure 8. Benchmark with variable thermo-physical properties - Percent difference in temperatures. 


\section{THERMO-PHYSICAL PROPERTIES}

The initial part of the work and analysis was based on thermo-physical properties that did not take into account the irradiation history of the various TRISO layers. The thermo-physical properties will be expanded and reported in a later document. The conductivities and specific heat capacities incorporated in this preliminary model are depicted in Figures 9 and 10, respectively.

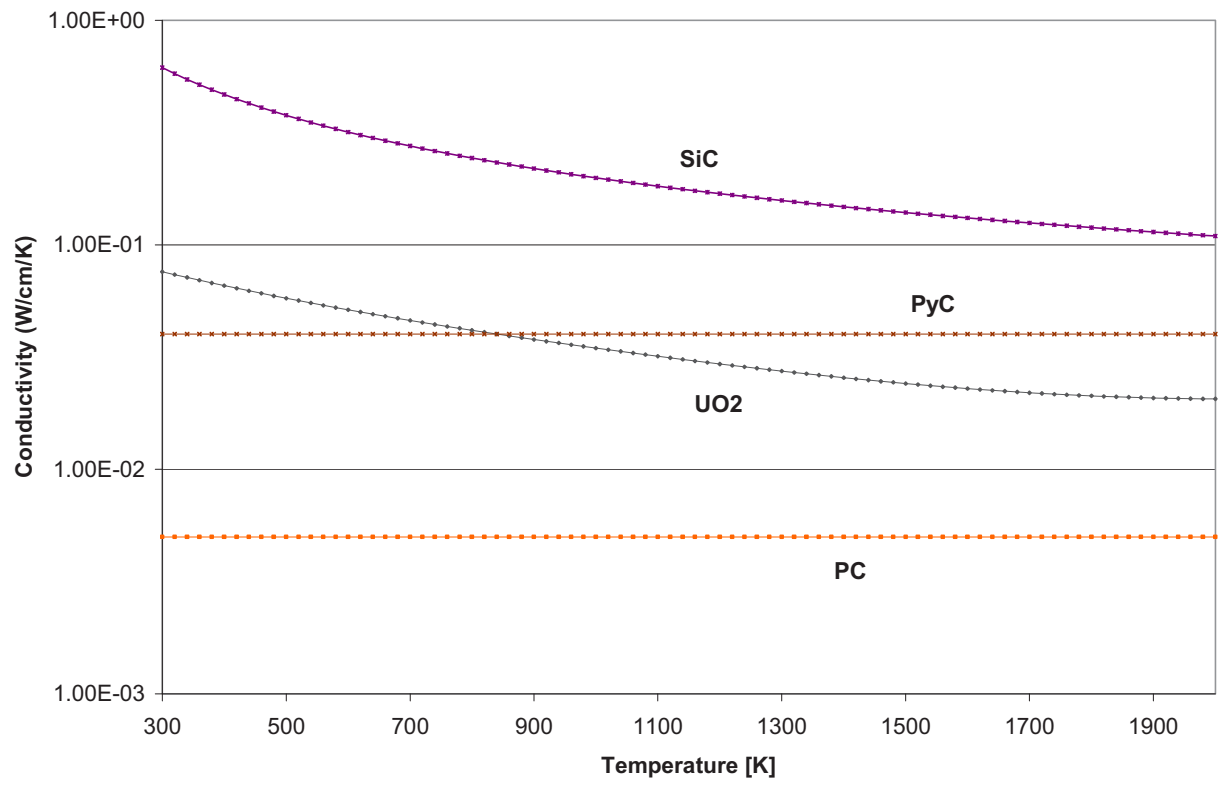

Figure 9. Thermal conductivities for the TRISO constituent layers.

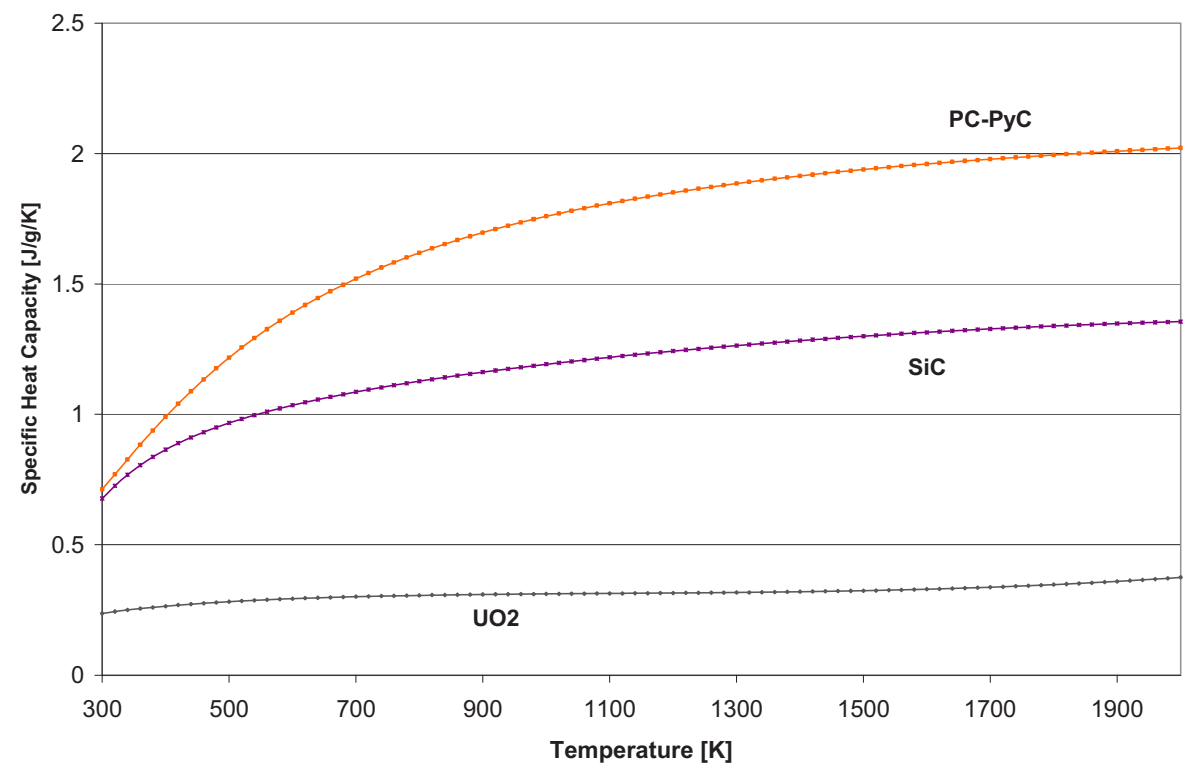

Figure 10. Specific heat capacities for the TRISO constituent layers. 


\section{INTEGRATION INTO THE COUPLED NEUTRONIC-TH SYSTEM}

Fuel models developed during this study are integrated in the coupled CYNOD-THERMIXKONVEK suite of codes. CYNOD (Hiruta 2008) is a semi-analytical nodal diffusion code based on a Green's function method with an implicit time-dependent scheme. CYNOD is coupled to the thermalhydraulics code THERMIX-KONVEK (Teuchert 1994), a two-dimensional (R-Z) quasi-static code developed by the KFA-Jülich, which contains correlations and material properties developed in the German PBR program. The homogeneous fuel model that is used in THERMIX is referred to as the quasi-static homogeneous (QSHo) model in this work.

Figure 11 shows a schematic of the coupling arrangement. Note that CYNOD has been coupled separately to THERMIX-KONVEK and RELAP5-3D. THERMIX-KONVEK is the thermal-hydraulic solver used in this work. The TRISO model is applied within each calculation cell and uses the cell average power density, renormalized to the kernel volume, as the heat generation source. The bulk graphite temperature from the thermal hydraulics calculation is used as the boundary condition at the OPyC-matrix interface. The interface thickness can be varied in order to adjust the location of the bulk graphite temperature within the pebble or compact.

The main idea behind this simplified modeling is to capture the behavior of the average TRISO particle in each calculation cell in a manner that is easily implemented in the current suite of codes to provide an initial analysis capability. The use of the Dirichlet condition with the bulk graphite temperature allows for a fast coupling of the heat transfer models to obtain a representative fuel temperature.

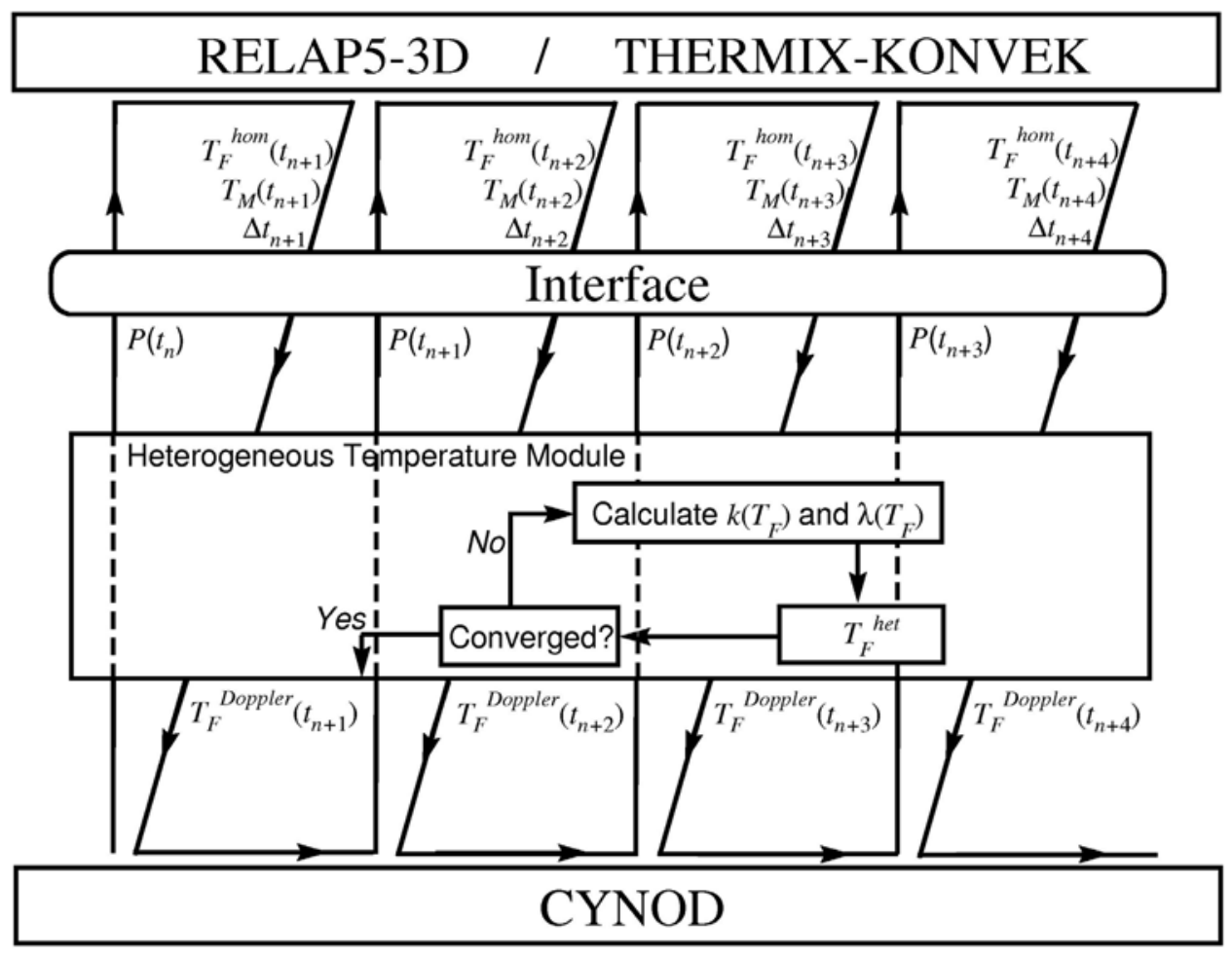

Figure 11. Coupling between the neutronic code CYNOD and thermal-hydraulics THERMIX-KONVEK (RELAP5-3D). 
Initially, a preliminary fuel temperature model was used to determine the potential benefits of developing a time-dependent kernel-level model. The steady-state equations from Section 4.1 were used for the model referred to as the quasi-static heterogeneous (QSHe) model. Given the success of the QSHe, the model developed in Section 4.2 (TDHe) and tested in Section 5 was incorporated in the coupled system. 


\section{EVALUATION OF THE INTEGRATED SYSTEM}

The total control rod ejection (TCRE) problem from the pebble bed modular reactor (PBMR) benchmark exercise was used to examine the potential benefits of the new method. This transient is included in the PBMR safety analysis report (SAR) as a design basis accident (DBA) (Strydom 2008), but there are currently no known initiators that could lead to this transient. Nevertheless, it serves to determine the capabilities of the explicit TRISO model in extreme cases. The results to the TCRE transient confirm the considerable improvement obtained by using explicit TRISO models over the predictions from the homogeneous model. Figure 12 shows how the prompt reactor feedback driven by the Doppler Effect has a more significant role than predicted by the original homogeneous THERMIX model. The calculated maximum powers are roughly 160, 26, and 30 times the nominal power for the QSHo, QSHe, and TDHe, respectively. Therefore, the QSHo model overestimates the reactor power by a factor of 130, while the QSHe underestimates the reactor power by a factor of 4 . In addition, the power curve indicates that the reactor period during the cool down is longer than previously predicted with the homogenous fuel region model.

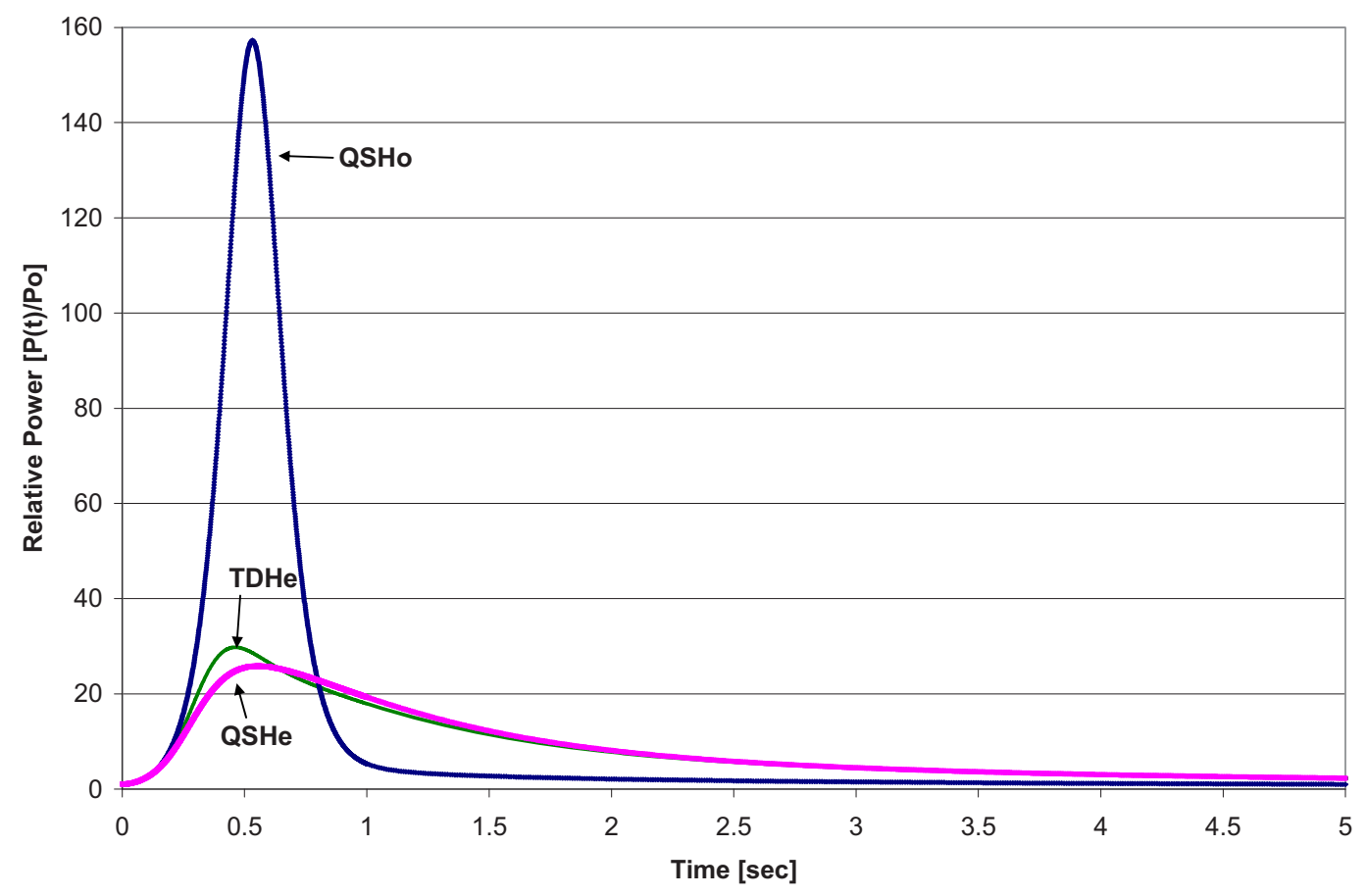

Figure 12. Relative reactor total power during the TCRE transient.

The maximum fission power calculated by the TDHe model is $11904 \mathrm{MW}$ and agrees well with the value of $11750 \mathrm{MW}$ obtained with TINTE (Strydom 2008). The power decays slightly faster in the TDHe model that in the TINTE analysis, but this is probably due to differences in thermo-physical properties of the model, since the TDHe analysis uses variable properties. The new model also reveals that the overall energy generation, and thus deposition, during the rod ejection transient is $12 \%$ lower than predicted by the unenhanced model.

Figure 13 shows that the average fuel temperature in the core reaches a slightly higher maximum, but at no time does it approach the nominal, allowable average fuel temperature of $1600^{\circ} \mathrm{C}$. The homogeneous model predicts a delayed moderator and fuel temperature rise that not only delay the 
Doppler feedback, resulting in a sharp power escalation, but it also produces nearly simultaneous Doppler and moderator feedback, yielding an equally fast drop in relative core power.

The maximum fuel temperature obtained with the TDHe is $1700^{\circ} \mathrm{C}$. A value of $1768^{\circ} \mathrm{C}$ is reported in the TINTE study, but it is based on a peaking model, which yields a higher temperature than the explicit TRISO model. The TINTE kernel model (Ubbink 2008) yields a kernel maximum temperature of $1691^{\circ} \mathrm{C}$. This shows that the INL results are in good agreement with the TINTE results for the PBMR benchmark case.

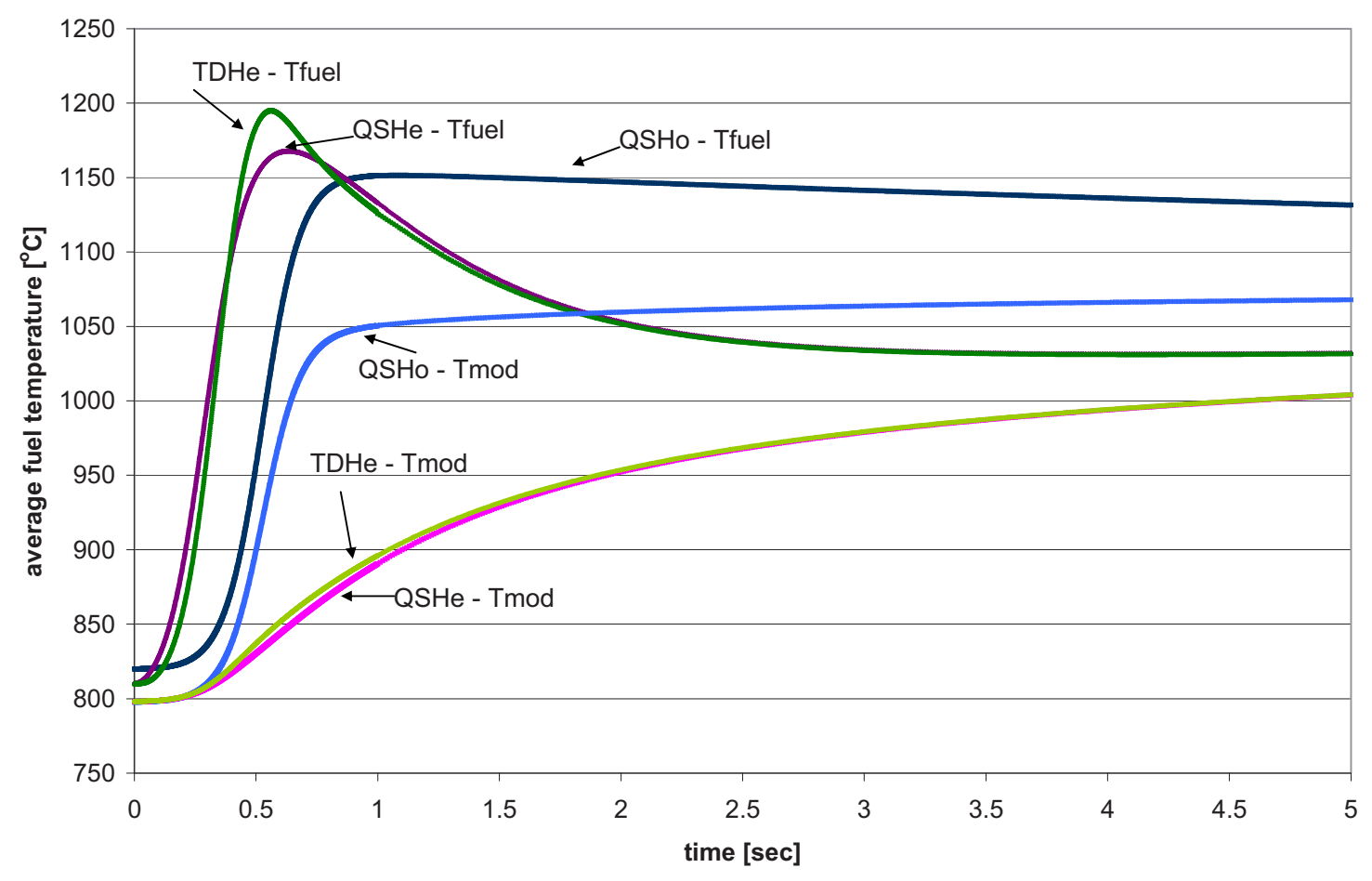

Figure 13. TCRE transient average temperatures. 


\section{CONCLUSIONS}

A simple and efficient way of calculating fuel temperature in TRISO-fueled reactors has been developed and implemented into the NGNP analysis package CYNOD-THERMIX-KONVEK. The model was benchmarked against analytic solutions and it performed well in code-to-code comparison versus Heating 7.3. The results show that both the quasi-static and the time-dependent heterogeneous models are a great improvement over the homogenous model for the study of the HTGR core behavior during fast transient. We have shown that a time-dependent heterogeneous model is able to capture dynamic effects that the quasi-static heterogeneous model is unable to capture. The fuel temperature calculations performed with the CYNOD-THERMIX-KONVEK suite are comparable to those of TINTE. 


\section{REFERENCES}

Childs, K. W., 2007, PSR-0199/05, "HEATING 7.3.”

Hiruta, H., et al., 2008, "CYNOD: a Neutronics Code for Pebble Bed Modular Reactor Coupled Transient Analysis," HTR.

Merrill, M. H., 1963, "Calculation Procedures for Evaluating Transients in Semihomogeneous Power Reactors," Reactor Kinetics and Control, Atomic Energy Commission Symposium 2.

“OECD/NEA/NSC PBMR Coupled Neutronic/Thermal Hydraulics Transient Benchmark: The PBMR-400 Core Design,” Draft 07 (2007).

Rademer, T., W. Bernnat, G. Lohnert, 2004, "Coupling of Neutronics and Thermal-Hydraulics Codes for the Simulation of Transients of Pebble Bed HTR Reactors," High Temperature Reactor Technology, Beijing, China, September 22-24, 2004.

Scherer, W., and E. Teuchert, 1989, “TINTE,” H. Gerwin, Nucl. Sci. Eng., Vol. 103, pp. 302-312.

Stainsby, R., et al., 2008, "Development of Local Heat Transfer Models for Safety Assessment of the Pebble Bed High Temperature Gas-Cooled Reactor Cores," High Temperature Reactor Technology, Washington, USA, September 28-October 1, 2008.

Stainsby, R., et al., 2008, "Development of Local Heat Transfer Models for the Safety Assessment of Prismatic Modular High Temperature Gas-Cooled Reactor Cores," High Temperature Reactor Technology, Washington, USA, September 28-October 1, 2008.

Strydom, G., 2008, “TINTE Transient Results for the OECD 400 MW PBMR Benchmark," ICAPP '08, Anaheim, CA, USA, June 8-12, 2008.

Teuchert, E., et al., 1994, V.S.O.P.('94) Computer Code System for Reactor Physics and Fuel Cycle Simulation - Input Manual and Comments, April 1994.

Ubbink, O., et al., 2008, "PBMR Fuel Kernel Model for the Prediction of Accurate Temperature Profiles," ICONE16, Orlando, Florida, USA, May 11-15, 2008. 


\section{Appendix A}

\section{Analytic Solution to the 1-D Spherical Steady State Heat Conduction Equation}




\section{Appendix A}

\section{Analytic Solution to the 1-D Spherical Steady State Heat Conduction Equation}

The 1-D spherical geometry steady-state heat conduction equation with constant volumetric heat generation rate is

$\frac{d^{2} T}{d r^{2}}+\frac{2}{r} \frac{d T}{d r}+\frac{q^{\prime \prime}}{k}=0$

Letting $\varphi=\frac{d T}{d r}$ and solving via the integrating factor yields

$\varphi(r)=\frac{d T}{d r}=-\frac{r q^{\prime \prime}}{3 k}+\frac{C_{1}}{r^{2}}$

Integrating again leads to

$T(r)=-\frac{r^{2} q^{\prime \prime}}{6 k}-\frac{C_{1}}{r}+C_{2}$

The temperature field in the innermost heated region is obtained by applying a Neumann $\left.\frac{d T}{d r}\right|_{r=0}=0$ and Dirichlet $T\left(r_{i+1}\right)=T_{i+1}$ boundary conditions to equation A-1

$T_{\text {heated }}(r)=\frac{q_{i}^{\prime \prime \prime}}{6 k_{i}}\left(r_{i+1}^{2}-r^{2}\right)+T_{i+1}$

The temperature field in non-heated regions is obtained by imposing to Equation AI-1 a non-zero heat flux at the lower boundary, $-\left.k_{1} \frac{d T}{d r}\right|_{r=r_{i}}=\frac{r_{f s} q_{1}^{\prime \prime}}{3}$, yielding

$T_{\text {unheated }}(r)=\frac{r_{f s}^{3} q_{1}^{\prime \prime \prime}}{3 k_{i}}\left(\frac{1}{r}-\frac{1}{r_{i-1}}\right)+T_{i-1}$

Where $r_{f s}$ is the fuel surface.

Average fuel temperatures in each region can be determined with 


$$
\langle T(r)\rangle=\frac{\int_{0}^{2 \pi} d \phi \int_{0}^{\pi} \sin \theta d \theta \int_{0}^{r_{1}} T(r) r^{2} d r}{\int_{0}^{2 \pi} d \phi \int_{0}^{\pi} \sin \theta d \theta \int_{0}^{r_{1}} r^{2} d r}
$$

Therefore, the average temperature in the fuel region under steady-state condition is

$$
\left\langle T_{\text {heated }}\right\rangle=\frac{r_{1}^{2}\left\langle q^{\prime \prime}\right\rangle}{15 k_{1}}+T_{2}
$$

The average temperature in the non-heated regions under steady-state conditions is

$$
\left\langle T_{\text {unheated }}\right\rangle=-q^{\prime \prime} r_{1}^{3} \frac{\left(r_{i-1}^{3}-3 r_{i-1} r_{i}^{2}+2 r_{i}^{3}\right)}{6 k_{i} r_{i-1}\left(r_{i}^{3}-r_{i-1}^{3}\right)}+T_{i-1}
$$

The node and cell average temperatures can be determined with Equations A-2 to A-5 given an outer boundary condition and a volumetric heat generation rate. 
Appendix B

\section{Numerical Solution to the 1-D Spherical Parabolic Heat Conduction Equation}




\section{Appendix B}

\section{Numerical Solution to the 1-D Spherical Parabolic Heat Conduction Equation}

Starting from the heat flux formulation of the conservation law

$-\nabla \cdot \vec{q}(r)+q_{t r}^{\prime \prime}(r)=\rho(r) C_{p}(r) \frac{\partial T(r, t)}{\partial t}$

Imposing a theta differencing scheme and the assumption that $\rho(r) C_{p}(r)$ do not change during the time step leads to

$-(1-\theta) \nabla \cdot \vec{q}^{n}+(1-\theta) q_{n}^{\prime \prime}-\theta \nabla \cdot \vec{q}^{n+1}+\theta q_{n+1}^{\prime \prime}=\rho(r) C_{p}(r) \frac{T^{n+1}-T^{n}}{t^{n+1}-t^{n}}$

Performing a heat balance by integrating the equation over the volume yields

$$
\begin{aligned}
& -(1-\theta) \iiint_{V_{i}} \nabla \cdot \vec{q}^{n} d V+(1-\theta) \iiint_{V_{i}} q_{n}^{\prime \prime} d V-\theta \iiint_{V_{i}} \nabla \cdot \vec{q}^{n+1} d V+\theta \iiint_{V_{i}} q_{n+1}^{\prime \prime \prime} d V \\
& =\iiint_{V_{i}} \rho(r) C_{p}(r) \frac{T^{n+1}-T^{n}}{t^{n+1}-t^{n}} d V
\end{aligned}
$$

Assuming the density and heat capacity to be homogeneous in the calculation cell leads to the following RHS for equation B-1

$R H S=\rho C_{p} \iiint_{V_{i}} \frac{T^{n+1}-T^{n}}{t^{n+1}-t^{n}} d V$

The node-wise temperatures can be related to cell averaged temperatures with

$\widetilde{T}_{i}^{n}=\frac{\iiint_{V_{i}} T^{n} d V}{\iiint_{V_{i}} d V}=\frac{\iiint_{V_{i}} T^{n} d V}{V_{i}}$

where $\quad V_{i}=\frac{r n_{i+1}^{3}-r n_{i}{ }^{3}}{3}$

Equation B-1 becomes

$$
\begin{aligned}
& -(1-\theta) \iiint_{V_{i}} \nabla \cdot \vec{q}^{n} d V+(1-\theta) \iiint_{V_{i}} q_{n}^{\prime \prime} d V-\theta \iiint_{V_{i}} \nabla \cdot \vec{q}^{n+1} d V+\theta \iiint_{V_{i}} q_{n+1}^{\prime \prime} d V \\
& =\widetilde{C}_{i}\left(\frac{\widetilde{T}_{i}^{n+1}-\widetilde{T}_{i}^{n}}{t^{n+1}-t^{n}}\right)
\end{aligned}
$$


Where the effective heat capacity is $\widetilde{C}_{i}=\rho C_{p} V_{i}$.

Using Gauss' divergence theorem and assuming constant heat generation in the volume we obtain

$$
\begin{aligned}
& -(1-\theta) A_{i+1} \vec{q}^{n}\left(r_{i+1}\right) \cdot \hat{n}_{i+1}+(1-\theta) A_{i} \vec{q}^{n}\left(r_{i}\right) \cdot \hat{n}_{i}-\theta A_{i+1} \vec{q}^{n+1}\left(r_{i+1}\right) \cdot \hat{n}_{i+1} \\
& +\theta A_{i} \vec{q}^{n+1}\left(r_{i}\right) \cdot \hat{n}_{i}+(1-\theta) q_{n}^{\prime \prime} V_{i}+\theta q_{n+1}^{\prime \prime} V_{i}=\left(\frac{\widetilde{T}_{i}^{n+1}-\widetilde{T}_{i}^{n}}{t^{n+1}-t^{n}}\right) \widetilde{C}_{i}
\end{aligned}
$$

Where

$\vec{q}^{n}\left(r_{i}\right)=-\left.k_{i} \frac{d T^{n}}{d r}\right|_{r_{i}}$ and $A_{i}=r_{i}^{2}$

Using a simple finite difference to approximate the temperature gradient leads to the following heat flux representations

$$
q_{i+\varepsilon}=-k_{i} \frac{\widetilde{T}_{i}-T_{i}}{\Delta r_{i} / 2} \text { and } q_{i-\varepsilon}=-k_{i-1} \frac{T_{i}-\widetilde{T}_{i-1}}{\Delta r_{i-1 / 2}}
$$

Imposing continuity of the heat flux with the approximate equations

$k_{i} \frac{\widetilde{T}_{i}-T_{i}}{\Delta r_{i}}=k_{i-1} \frac{T_{i}-\widetilde{T}_{i-1}}{\Delta r_{i-1}}$

$\frac{k_{i}}{\Delta r_{i}}\left(\widetilde{T}_{i}-T_{i}\right)=\frac{k_{i-1}}{\Delta r_{i-1}}\left(T_{i}-\widetilde{T}_{i-1}\right)$

$\frac{k_{i}}{\Delta r_{i}} \widetilde{T}_{i}-\frac{k_{i}}{\Delta r_{i}} T_{i}=\frac{k_{i-1}}{\Delta r_{i-1}} T_{i}-\frac{k_{i-1}}{\Delta r_{i-1}} \widetilde{T}_{i-1}$

This enables the interpolation of one nodal temperature value from two adjacent cell-averaged values $T_{i}=\frac{\frac{k_{i-1}}{\Delta r_{i-1}} \widetilde{T}_{i-1}+\frac{k_{i}}{\Delta r_{i}} \widetilde{T}_{i}}{\frac{k_{i}}{\Delta r_{i}}+\frac{k_{i-1}}{\Delta r_{i-1}}}$

Inserting this temperature interpolator in the definition of the heat flux

$q_{i}=-\frac{2 k_{i}}{\Delta r_{i}} \widetilde{T}_{i}+\frac{2 k_{i}}{\Delta r_{i}} T_{i}$ 
$q_{i}=-\frac{2 k_{i}}{\Delta r_{i}} \widetilde{T}_{i}+\frac{2 k_{i}}{\Delta r_{i}} \frac{\frac{k_{i-1}}{\Delta r_{i-1}} \widetilde{T}_{i-1}+\frac{k_{i}}{\Delta r_{i}} \widetilde{T}_{i}}{\frac{k_{i}}{\Delta r_{i}}+\frac{k_{i-1}}{\Delta r_{i-1}}}$

Simplifying the equation leads to

$q_{i}=-\frac{2 k_{i} k_{i-1}}{\left(\Delta r_{i-1} k_{i}+\Delta r_{i} k_{i-1}\right)} \widetilde{T}_{i}+\frac{2 k_{i} k_{i-1}}{\left(\Delta r_{i-1} k_{i}+\Delta r_{i} k_{i-1}\right)} \widetilde{T}_{i-1}$

Therefore, we have the following discretizations for the heat flux at the boundaries

$\vec{q}_{i}^{n}=-\frac{2 k_{i} k_{i-1}}{\left(\Delta r_{i-1} k_{i}+\Delta r_{i} k_{i-1}\right)} \widetilde{T}_{i}+\frac{2 k_{i} k_{i-1}}{\left(\Delta r_{i-1} k_{i}+\Delta r_{i} k_{i-1}\right)} \widetilde{T}_{i-1}$
${\overrightarrow{q_{i+1}}}^{n}=-\frac{2 k_{i+1} k_{i}}{\left(\Delta r_{i} k_{i+1}+\Delta r_{i+1} k_{i}\right)} \widetilde{T}_{i+1}+\frac{2 k_{i+1} k_{i}}{\left(\Delta r_{i} k_{i+1}+\Delta r_{i+1} k_{i}\right)} \widetilde{T}_{i}$

Inserting Equations B-4 and B-5 in the heat balance equation B-3 leads to the final discretization

$\theta \frac{2 A_{i+1} k_{i+1} k_{i}}{\left(\Delta r_{i} k_{i+1}+\Delta r_{i+1} k_{i}\right)} \widetilde{T}_{i+1}^{n+1}-\theta\left(\frac{2 A_{i+1} k_{i+1} k_{i}}{\left(\Delta r_{i} k_{i+1}+\Delta r_{i+1} k_{i}\right)}+\frac{2 A_{i} k_{i} k_{i-1}}{\left(\Delta r_{i-1} k_{i}+\Delta r_{i} k_{i-1}\right)}\right) \widetilde{T}_{i}^{n+1}$

$+\theta \frac{2 A_{i} k_{i} k_{i-1}}{\left(\Delta r_{i-1} k_{i}+\Delta r_{i} k_{i-1}\right)} \widetilde{T}_{i-1}^{n+1}=\left(\frac{\widetilde{T}_{i}^{n+1}-\widetilde{T}_{i}^{n}}{\Delta t^{n+1}}\right) \widetilde{C}_{i}$

$-(1-\theta) \frac{2 A_{i+1} k_{i+1} k_{i}}{\left(\Delta r_{i} k_{i+1}+\Delta r_{i+1} k_{i}\right)} \widetilde{T}_{i+1}^{n}+(1-\theta)\left(\frac{2 A_{i+1} k_{i+1} k_{i}}{\left(\Delta r_{i} k_{i+1}+\Delta r_{i+1} k_{i}\right)}+\frac{2 A_{i} k_{i} k_{i-1}}{\left(\Delta r_{i-1} k_{i}+\Delta r_{i} k_{i-1}\right)}\right) \widetilde{T}_{i}^{n}$

$-(1-\theta) \frac{2 A_{i} k_{i} k_{i-1}}{\left(\Delta r_{i-1} k_{i}+\Delta r_{i} k_{i-1}\right)} \widetilde{T}_{i-1}^{n}$

$-\left[(1-\theta) q_{n}^{\prime \prime \prime}+\theta q_{n+1}^{\prime \prime \prime}\right] Y_{i}$

Rearranging yields a three point formulation

$a_{i, i+1} \widetilde{T}_{i+1}^{n+1}+a_{i, i} \widetilde{T}_{i}^{n+1}+a_{i, i-1} \widetilde{T}_{i-1}^{n+1}=\widehat{Q}_{i}^{\cdots \prime}+b_{i, i+1} \widetilde{T}_{i+1}^{n}+b_{i, i} \widetilde{T}_{i}^{n}+b_{i, i-1} \widetilde{T}_{i-1}^{n}$

With the coefficients

$$
\begin{aligned}
& a_{i, i+1}=\theta \frac{2 A_{i+1} k_{i+1} k_{i}}{\left(\Delta r_{i} k_{i+1}+\Delta r_{i+1} k_{i}\right)} \\
& a_{i, i}=-\left[\theta\left(\frac{2 A_{i+1} k_{i+1} k_{i}}{\left(\Delta r_{i} k_{i+1}+\Delta r_{i+1} k_{i}\right)}+\frac{2 A_{i} k_{i} k_{i-1}}{\left(\Delta r_{i-1} k_{i}+\Delta r_{i} k_{i-1}\right)}\right)+\frac{\widetilde{C}_{i}}{\Delta t^{n+1}}\right]
\end{aligned}
$$




$$
\begin{aligned}
& a_{i, i-1}=\theta \frac{2 A_{i} k_{i} k_{i-1}}{\left(\Delta r_{i-1} k_{i}+\Delta r_{i} k_{i-1}\right)} \\
& b_{i, i+1}=-(1-\theta) \frac{2 A_{i+1} k_{i+1} k_{i}}{\left(\Delta r_{i} k_{i+1}+\Delta r_{i+1} k_{i}\right)} \\
& b_{i, i}=\left[(1-\theta)\left(\frac{2 A_{i+1} k_{i+1} k_{i}}{\left(\Delta r_{i} k_{i+1}+\Delta r_{i+1} k_{i}\right)}+\frac{2 A_{i} k_{i} k_{i-1}}{\left(\Delta r_{i-1} k_{i}+\Delta r_{i} k_{i-1}\right)}\right)-\frac{\widetilde{C}_{i}}{\Delta t^{n+1}}\right] \\
& b_{i, i-1}=-(1-\theta) \frac{2 A_{i} k_{i} k_{i-1}}{\left(\Delta r_{i-1} k_{i}+\Delta r_{i} k_{i-1}\right)} \\
& \widehat{Q}_{i}^{\prime \prime}=-\left[(1-\theta) q_{n}^{\prime \prime}+\theta q_{n+1}^{\prime \prime}\right]_{i}
\end{aligned}
$$

The resulting matrix is symmetric and can be easily solved with direct or iterative methods.

The theta differencing scheme allows the variation of the time discretization from a first order implicit method $\theta=1$ to a first order explicit method $\theta=0$. Second order accuracy is achieved with the Crank-Nicholson method $\theta=0.5$.

\section{Imposition of Boundary Conditions}

An interior Neumann boundary condition leads to the following equation for the innermost cell

$$
\begin{aligned}
& -\left[\theta \frac{2 A_{2} k_{1} k_{2}}{\left(k_{2} \Delta r_{1}+\Delta r_{2} k_{1}\right)}+\frac{\widetilde{C}_{1}}{t^{n+1}-t^{n}}\right] \widetilde{T}_{1}^{n+1}+\theta \frac{2 A_{2} k_{1} k_{2}}{\left(k_{2} \Delta r_{1}+\Delta r_{2} k_{1}\right)} \widetilde{T}_{2}^{n+1}=-\left[(1-\theta) q_{n}^{\prime \prime \prime}+\theta q_{n+1}^{\prime \prime \prime}\right]_{i_{1}} \\
& -(1-\theta) \frac{2 A_{2} k_{1} k_{2}}{\left(k_{2} \Delta r_{1}+\Delta r_{2} k_{1}\right)}\left(\widetilde{T}_{2}^{n}-\widetilde{T}_{1}^{n}\right)-\frac{\widetilde{C}_{1}}{\Delta t^{n+1}} \widetilde{T}_{1}^{n}
\end{aligned}
$$

A mixed condition on the outer boundary can be readily built with $\alpha= \begin{cases}1 & \text { Dirichlet B.C. } \\ 0 & \text { Neumann B.C }\end{cases}$

$$
\begin{aligned}
& {\left[\theta\left(-\alpha \frac{k_{N} 2 A_{N+1}}{\Delta r_{N}}-\frac{k_{N-1} k_{N} 2 A_{N}}{\left(k_{N} \Delta r_{N-1}+\Delta r_{N} k_{N-1}\right)}\right)-\frac{\widetilde{C}_{N}}{t^{n+1}-t^{n}}\right] \widetilde{T}_{N}^{n+1}+\theta \frac{k_{N-1} k_{N} 2 A_{N}}{\left(k_{N} \Delta r_{N-1}+\Delta r_{N} k_{N-1}\right)} \widetilde{T}_{N-1}^{n+1}=-\left[(1-\theta) q_{n}^{\prime \prime \prime}+\theta q_{n+1}^{\prime \prime}\right] V_{i}} \\
& -\alpha \frac{k_{N} 2 A_{N+1}}{\Delta r_{N}} T_{g}+\left[(1-\theta)\left(\alpha \frac{k_{N} 2 A_{N+1}}{\Delta r_{N}}+\frac{k_{N-1} k_{N} 2 A_{N}}{\left(k_{N} \Delta r_{N-1}+\Delta r_{N} k_{N-1}\right)}\right)-\frac{\widetilde{C}_{N}}{t^{n+1}-t^{n}}\right] \widetilde{T}_{N}^{n}-(1-\theta) \frac{k_{N-1} k_{N} 2 A_{N}}{\left(k_{N} \Delta r_{N-1}+\Delta r_{N} k_{N-1}\right)} \widetilde{T}_{N-1}^{n}
\end{aligned}
$$




\section{Appendix C}

\section{Analytic Solution to the 1-D Spherical Parabolic Heat Conduction Equation}




\section{Appendix C}

\section{Analytic Solution to the 1-D Spherical Parabolic Heat Conduction Equation}

The purpose of this appendix is to find an analytic solution to the parabolic heat conduction equation

that can be used in the benchmarking of the numerical solution. To simplify the task, a one-region model with constant thermo-physical properties is employed.

The parabolic heat conduction equation with a heat source is:

$\frac{\partial^{2} T(r, t)}{\partial r^{2}}+\frac{2}{r} \frac{\partial T(r, t)}{\partial r}+\frac{q_{t r}^{\prime \prime}(r, t)}{k}=\frac{1}{\alpha} \frac{\partial T(r, t)}{\partial t} \quad 0<r<R$ for $\mathrm{t}>0$

Where $\alpha=\frac{k}{\rho C_{p}}$ (thermal diffusivity)

With boundary conditions

$T(0, t)=$ finite

$T(R, t)=T_{\text {graphite }}$

Initial Condition

$T(r)=T_{\text {graphite }}$ at $\mathrm{t}=0$

One can generate a homogenous boundary condition for the problem with a simple change of variables

$\varphi(r, t)=T(r, t)-T_{\text {graphite }}$

$\frac{\partial^{2} \varphi(r, t)}{\partial r^{2}}+\frac{2}{r} \frac{\partial \varphi(r, t)}{\partial r}+\frac{q_{t r}^{\prime \prime}(r, t)}{k}=\frac{1}{\alpha} \frac{\partial \varphi(r, t)}{\partial t}$

$\varphi(0, t)=$ finite

$\varphi(R, t)=0$

Initial Condition

$\varphi(r)=0$ at $\mathrm{t}=0$

Now one can solve the time-dependent heat generation problem with the Green's function method. Consider the homogeneous problem:

$\frac{\partial^{2} \theta(r, t)}{\partial r^{2}}+\frac{2}{r} \frac{\partial \theta(r, t)}{\partial r}=\frac{1}{\alpha} \frac{\partial \theta(r, t)}{\partial t}$ 
$\theta(0, t)=$ finite for $\mathrm{t} \geq 0$

$\theta(R, t)=0$ for $\mathrm{t} \geq 0$

$\theta(r, t)=F(r)$ for $\mathrm{t}=0$

Let $U(r, t)=r \theta(r, t)$ to obtain

$\frac{\partial^{2} U(r, t)}{\partial r^{2}}=\frac{1}{\alpha} \frac{\partial U(r, t)}{\partial t}$

$\mathrm{U}(\mathrm{r}=0)=\mathrm{U}(\mathrm{r}=\mathrm{R})=0$ or $\mathrm{t} \geq 0$

and

$\mathrm{U}(\mathrm{r}, \mathrm{t})=\mathrm{rF}(\mathrm{r})$ for $\mathrm{t}=0$

The general solution to Equation C-5 is

$U(r, t)=e^{-\alpha \beta_{n}^{2} t}\left[A_{n} \sin \left(\beta_{n} r\right)+B_{n} \cos \left(\beta_{n} r\right)\right]$

Since $\mathrm{U}_{\mathrm{h}}(\mathrm{r}=0)=\mathrm{U}_{\mathrm{h}}(\mathrm{r}=\mathrm{R})=0$

$U(0, t)=e^{-\alpha \beta_{n}^{2} t}\left[A_{n} \sin \left(\beta_{n} 0\right)+B_{n} \cos \left(\beta_{n} 0\right)\right]=0$

$B_{n}=0$

And

$U(R, t)=\sin \left(\beta_{n} R\right)=0$

Therefore, the eigen values are $\beta_{n}=\frac{n \pi}{R}$

$U(r, t)=\sum_{n=1}^{\infty} A_{n} e^{-\alpha \beta_{n}^{2} t} \sin \left(\beta_{n} r\right)$

Since

$U(r, t)=r \theta(r, t)$

$\theta(r, t)=\sum_{n=1}^{\infty} A_{n} e^{-\alpha \beta_{n}^{2} t} \frac{\sin \left(\beta_{n} r\right)}{r}$

Applying the I.C. $\theta(r, t)=F(r) \mathrm{t}=0$

$\theta(r, 0)=\sum_{n=1}^{\infty} A_{n} \frac{\sin \left(\beta_{n} r\right)}{r}=F(r)$ 


$$
\begin{aligned}
& \sum_{n=1}^{\infty} A_{n} \int_{0}^{R} r^{\prime 2} d r^{\prime} \frac{\sin \left(\beta_{n} r^{\prime}\right)}{r^{\prime}} \frac{\sin \left(\beta_{n} r^{\prime}\right)}{r^{\prime}}=\int_{0}^{R} r^{\prime 2} d r^{\prime} F\left(r^{\prime}\right) \frac{\sin \left(\beta_{n} r^{\prime}\right)}{r^{\prime}} \\
& A_{n}=\frac{2}{R} \int_{0}^{R} r^{\prime 2} d r^{\prime} F\left(r^{\prime}\right) \frac{\sin \left(\beta_{n} r^{\prime}\right)}{r^{\prime}}
\end{aligned}
$$

Then

$$
\theta(r, t)=\int_{0}^{R} r^{\prime 2}\left[\frac{2}{R r^{\prime} r} \sum_{n=1}^{\infty} e^{-\alpha \beta_{n}^{2} t} \sin \left(\beta_{n} r^{\prime}\right) \sin \left(\beta_{n} r\right)\right] F\left(r^{\prime}\right) d r^{\prime}
$$

Therefore, the Green's function is

$G\left(r, t \mid r^{\prime}, \tau\right)=\frac{2}{R r^{\prime} r} \sum_{n=1}^{\infty} e^{-\alpha \beta_{n}^{2}(t-\tau)} \sin \left(\beta_{n} r^{\prime}\right) \sin \left(\beta_{n} r\right)$

The solution to the non-homogeneous problem is obtained from

$\varphi(r, t)=\frac{\alpha}{k} \int_{0}^{t} d \tau \int_{0}^{R} r^{\prime 2} G\left(r, t \mid r^{\prime}, \tau\right) q^{\prime \prime \prime}(r, \tau) d r^{\prime}$

Assuming a spatially independent heat generation rate leads to

$\varphi(r, t)=\frac{\alpha}{k} \frac{2}{R r} \sum_{n=1}^{\infty} \sin \left(\beta_{n} r\right) e^{-\alpha \beta_{n}^{2} t} \int_{0}^{R} r^{\prime} \sin \left(\beta_{n} r^{\prime}\right) d r^{\prime} \int_{0}^{t} q^{\prime \prime \prime}(\tau) e^{\alpha \beta_{n}^{2} \tau} d \tau$

Carrying out the spatial integration gives the temperature field

$\varphi(r, t)=-\frac{\alpha}{k} \frac{2 R}{r} \sum_{n=1}^{\infty} \frac{(-1)^{n}}{n \pi} \sin \left(\beta_{n} r\right) e^{-\alpha \beta_{n}^{2} t} \int_{0}^{t} q^{\prime \prime \prime}(\tau) e^{\alpha \beta_{n}^{2} \tau} d \tau$

\section{Case 1. Constant Heat Generation}

The time integration in equation C-7 under the assumption of constant heat generation becomes

$$
\varphi(r, t)=-q^{\prime \prime \prime} \frac{2 R^{3}}{k} \sum_{n=1}^{\infty} \frac{(-1)^{n}}{(n \pi)^{3}} \frac{\sin \left(\beta_{n} r\right)}{r}\left(1-e^{-\alpha \beta_{n}^{2} t}\right)
$$

\section{Case 2. Linear Heat Generation}

Assuming a linear heat generation rate of the form $q^{\prime \prime \prime}(t)=a_{1}+a_{2} t$ and carrying out the time integration

$$
\varphi(r, t)=-\frac{\alpha}{k} 2 R \sum_{n=1}^{\infty} \frac{(-1)^{n}}{n \pi} \frac{\sin \left(\beta_{n} r\right)}{r} e^{-\alpha \beta_{n}^{2} t}\left(\int_{0}^{t} a_{1} e^{\alpha \beta_{n}^{2} \tau} d \tau+\int_{0}^{t} a_{2} \tau e^{\alpha \beta_{n}^{2} \tau} d \tau\right)
$$




$$
\varphi(r, t)=-\frac{\alpha}{k} 2 R \sum_{n=1}^{\infty} \frac{(-1)^{n}}{n \pi} \frac{\sin \left(\beta_{n} r\right)}{r} e^{-\alpha \beta_{n}^{2} t}\left(\frac{a_{1}}{\alpha \beta_{n}^{2}}\left(e^{\alpha \beta_{n}^{2} t}-1\right)+a_{2}\left(\frac{\alpha \beta_{n}^{2} t e^{\alpha \beta_{n}^{2} t}-e^{\alpha \beta_{n}^{2} t}+1}{\alpha^{2} \beta_{n}^{4}}\right)\right)
$$

Simplifying the above equation yields the temperature field equation under linear heat generation

$$
\varphi(r, t)=-\frac{\alpha}{k} 2 R \sum_{n=1}^{\infty} \frac{(-1)^{n}}{n \pi} \frac{\sin \left(\beta_{n} r\right)}{r}\left(\frac{a_{1}}{\alpha \beta_{n}^{2}}\left(1-e^{-\alpha \beta_{n}^{2} t}\right)+a_{2}\left(\frac{\alpha \beta_{n}^{2} t-1+e^{-\alpha \beta_{n}^{2} t}}{\alpha^{2} \beta_{n}^{4}}\right)\right)
$$

The average temperature is

$\langle\varphi(t)\rangle=\frac{\int_{r_{i}}^{r_{i+1}} \varphi(r, t) r^{2} d r}{\int_{r_{i}}^{r_{i+1}} r^{2} d r}=$

$\frac{-\frac{2 \alpha R}{k} \sum_{n=1}^{\infty} \frac{(-1)^{n}}{n \pi}\left(\frac{a_{1}}{\alpha \beta_{n}^{2}}\left(1-e^{-\alpha \beta_{n}^{2} t}\right)+a_{2}\left(\frac{\alpha \beta_{n}^{2} t-1+e^{-\alpha \beta_{n}^{2} t}}{\alpha^{2} \beta_{n}^{4}}\right)\right) \frac{\left[\sin \left(\beta_{n} r_{i+1}\right)-\beta_{n} r_{i+1} \cos \left(\beta_{n} r_{i+1}\right)-\sin \left(\beta_{n} r_{i}\right)+\beta_{n} r_{i} \cos \left(\beta_{n} r_{i}\right)\right]}{\beta_{n}^{2}}}{\int_{r_{i}}^{r_{i+1}} r^{2} d r}$

Case 3. Exponential heat source

Under the assumption of exponential heat generation rate $q^{\prime \prime \prime}\left(\tau_{0}+\Delta \tau\right)=q^{\prime \prime \prime}\left(\tau_{0}\right) e^{\omega_{1}\left(\tau_{0}+\Delta \tau\right)}$.

The value of the exponential factor is

$$
\frac{q^{\prime \prime \prime}\left(\tau_{0}+\Delta \tau\right)}{q^{\prime \prime \prime}\left(\tau_{0}\right)}=e^{\omega_{1}\left(\tau_{0}+\Delta \tau\right)} \text { and } \omega_{1}=\frac{\ln \left(q^{\prime \prime \prime}\left(\tau_{0}+\Delta \tau\right)\right)-\ln \left(q^{\prime \prime \prime}\left(\tau_{0}\right)\right)}{\left(\tau_{0}+\Delta \tau\right)}
$$

The generalized time-dependent source for a given time step is

$$
\varphi(r, t)=-q^{\prime \prime \prime}\left(\tau_{0}\right) \frac{\alpha}{k} \frac{2 R}{r} \sum_{n=1}^{\infty} \frac{(-1)^{n}}{n \pi} \sin \left(\beta_{n} r\right) e^{-\alpha \beta_{n}^{2} t} \int_{0}^{t} e^{\left(\omega_{1}+\alpha \beta_{n}^{2}\right) \tau} d \tau
$$

The temperature field equation under exponential heat generation rate is

$$
\begin{aligned}
& \varphi(r, t)=-q^{\prime \prime \prime}\left(\tau_{0}\right) \frac{\alpha}{k} 2 R \sum_{n=1}^{\infty} \frac{(-1)^{n}}{n \pi} \frac{\sin \left(\beta_{n} r\right)}{r} \frac{\left(e^{\omega_{1} t}-e^{-\alpha \beta_{n}^{2} t}\right)}{\left(\omega_{1}+\alpha \beta_{n}^{2}\right)} \\
& \langle\varphi(t)\rangle=\frac{\int_{r_{i}}^{r_{i+1}} \varphi(r, t) r^{2} d r}{\int_{r_{i}}^{r_{i+1}} r^{2} d r}=\frac{-q^{\prime \prime \prime}\left(\tau_{0}\right) \frac{\alpha}{k} 2 R \sum_{n=1}^{\infty} \frac{(-1)^{n}}{n \pi} \frac{\left(e^{\omega_{1} t}-e^{-\alpha \beta_{n}^{2} t}\right)}{\left(\omega_{1}+\alpha \beta_{n}^{2}\right)} \int_{r_{i}}^{r_{i+1}} \sin \left(\beta_{n} r\right) r d r}{\int_{r_{i}}^{r_{i+1}} r^{2} d r}
\end{aligned}
$$

32 


$$
\langle\varphi(t)\rangle=\frac{-q^{\prime \prime \prime}\left(\tau_{0}\right) \frac{\alpha}{k} 2 R \sum_{n=1}^{\infty} \frac{(-1)^{n}}{n \pi} \frac{\left(e^{\omega_{1} t}-e^{-\alpha \beta_{n}^{2} t}\right)}{\left(\omega_{1}+\alpha \beta_{n}^{2}\right)} \frac{\left[\sin \left(\beta_{n} r_{i+1}\right)-\beta_{n} r_{i+1} \cos \left(\beta_{n} r_{i+1}\right)-\sin \left(\beta_{n} r_{i}\right)+\beta_{n} r_{i} \cos \left(\beta_{n} r_{i}\right)\right]}{\beta_{n}^{2}}}{\int_{r_{i}}^{r_{i+1}} r^{2} d r}
$$

